\title{
High resolution imaging with multilayer telescopes: resolution performance of the MSSTA II Telescopes
}

\author{
D. S. Martínez-Galarce ${ }^{\mathrm{a}, *}$; A. B. C. Walker, II ${ }^{\text {b,c }}$; D. B. Gore ${ }^{\text {d}}$; C. C. Kankelborg ${ }^{\text {e. }}$ R. B. Hoover ${ }^{\text {f }}$; \\ T. W. Barbee, II ${ }^{\text {g; P. F. X. Boerner }}{ }^{\text {b }}$ \\ ${ }^{a}$ W. W. Hansen Experimental Physics Laboratory; Stanford University; Stanford, CA 94305-4085 USA \\ ${ }^{b}$ Department of Physics; Stanford University; Stanford, CA 94305-4060 USA \\ ${ }^{c}$ Department of Applied Physics; Stanford University; Stanford, CA 94305-4090 USA \\ ${ }^{\mathrm{d}}$ Department of Physics; University of Alabama; Birmingham, AL 35294-1170 USA

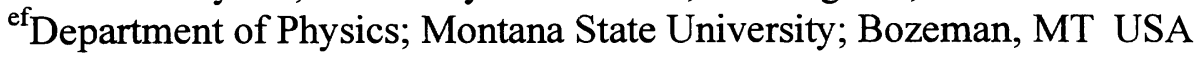 \\ ${ }^{f}$ Space Science Laboratory, ES-52; NASA-Marshall Space Flight Center; Huntsville, AL 35812 USA \\ ${ }^{g}$ Lawrence Livermore National Lab.; P. O. Box 808; Livermore, CA 94550 USA
}

\begin{abstract}
The Multi-Spectral Solar Telescope Array (MSSTA) is a sounding rocket-borne observatory composed of a set of normalincidence multilayer-coated telescopes that obtained selected bandpass spectroheliograms $(44 \AA-1550 \AA)$ of the Solar atmosphere. These spectroheliograms were recorded on specially fabricated XUV and FUV 70mm Kodak film. Rocket launches of this instrument payload took place in 1991 (MSSTA I) and 1994 (MSSTA II) at the White Sands Missile Test Range in New Mexico, sponsored by the NASA sounding rocket experiment program. Immediately prior to the 1994 launch, visible light focusing tests of each telescope were performed in-situ using a 1951 Standard Air Force High Resolution Testtarget, to measure optical resolution performance. We determined that the MSSTA II telescopes performed at diffractionlimited resolutions down to 0.70 arc-second at visible wavelengths. Based on these measurements, we calculated an upperbound to the focusing errors that incorporate the sum of all uncorrelated system resolution errors that affect resolution performance. Coupling these upper-bound estimates with the in-band diffraction limits, surface scattering errors and payload pointing jitter, we demonstrate that eleven of nineteen MSSTA II telescopes - having negligible figures of focus errors in comparison to the corresponding visible diffraction limits - performed at sub arc-second resolution at their operational FUV/EUV/XUV wavelengths during flight. We estimate the in-band performance down to $0.14 \pm 0.08$ second of arc.
\end{abstract}

Keywords: $\quad$ multilayer optics, space optics, solar chromosphere, solar corona, spectroheliograph, FUV-XUV imaging.

\section{INTRODUCTION}

The use of Multilayer optics for high resolution imaging ( $\leq 1$ arcsecond) at FUV, EUV and soft x-ray wavelengths is a technique that is now standard in spectroheliograph observations of the solar atmosphere. This technology has been pioneered and independently developed by two main research consortia whose initial observations were performed on sounding rocket flights on 23 October 1987, led by Walker et al. $;^{1}$ and 23 June 1988, led by Golub et al. ${ }^{2}$, 3 These two groups were the first to record $\sim 1$ arc-second narrow bandpass spectroheliograms using normal-incidence, multilayer optics. The thin film deposition techniques of multilayers have been developed independently by Spiller, ${ }^{4-6}$ and Barbee. ${ }^{7,8}$ In the 1987 flight, Walker et al. reported an in-band resolution of $\sim 1.1$ arc-second performance of a $173 \AA$ Cassegrain telescope on the Stanford-Marshall Space Flight Center Spectroheliograph (Stanford-MSFC-LLNL) payload. In a follow-on flight of the Normal-incidence X-ray Telescope (NIXT) in 1989 by Golub et al. ${ }^{9}$ (Harvard-Smithsonian-IBM), a resolution performance of $\sim 0.75$ arc-second was reported with the $63.5 \AA$ Ritchey-Chrétien recorded observations. In the former, it was reported that resolution performance of the $173 \AA$ Cassegrain was limited to optic figure errors; while in the latter, film resolution measurements at soft x-ray wavelengths were considered the limitation on resolution. In both cases, a detailed analysis or

\footnotetext{
*Correspondance: Email: denmart@seyfert.stanford.edu: Tel.: (650) 725-0448; Fax.: (650) 723-1487.
} 
measurement of the in-band point spread function was not performed as it is a difficult and expensive measurement to conduct properly.

Prior to the initial flight of multilayer telescopes in wavelength bands centered at $173 \AA$ and $256 \AA$, the StanfordMSFC-LLNL consortium carried out a resolution test on a specially prepared Cassegrain telescope at $44.3 \AA$ (identical to the $173 \AA$ Cassegrain) using multilayer mirrors designed to reflect the $\mathrm{C} \mathrm{K} \alpha$ line. These tests were carried out in the MSFC Xray Calibration Facility (XRCF) by Lindblom et al., 10,11 who showed that the resolution of the multilayer mirrors was limited by the aberrations of the mirror substrates and also because of a mechanical deformation of the primary mirror introduced by its holding mechanism. Although efficiency tests have been carried out on all multilayer optical systems subsequently flown, the difficulty and expense of fabricating optical testing systems that can image the characteristic soft $\mathrm{x}$ ray and EUV wavelengths that can be generated with laboratory sources, has precluded in-band resolution testing. Instead, we have carried out resolution tests at optical wavelengths using laser interferometric techniques to verify the optical figure, alignment and spatial frequency response of our flight telescopes. Coupled with these interferometric tests, visible light imaging measurements using a calibrated, standard high resolution test-target were also performed and an estimate of the focusing errors calculated to determine an upper-bound of the in-band resolution performance for each of the multilayer telescopes which comprised the second flight of the Multi-Spectral Solar Telescope Array (MSSTA II). In the current analysis, we present these estimates and describe the experimental approach that was carried out (prior to launch) to determine them.

\subsection{MSSTA}

The Multi-Spectral Solar Telescope Array (MSSTA) is a sounding rocket-borne observatory designed to obtain high spatial resolution spectroheliograms of the Sun at selected soft X-ray (XUV) \{44.1-93.9 $\}$, Extreme Ultraviolet (EUV) $\{150-335 \AA\}$, and Far Ultraviolet (FUV) $\{1215.6$ and $1550 \AA\}$ wavelengths. Images in these bandpasses were recorded on specially fabricated XUV $(X U V-100)$ and FUV (649) film manufactured by Kodak. Two sounding rocket experiments were performed with this payload, the first flight (36.049) was at 1905 UT on May 13, 1991, (MSSTA I) and the second flight (36.091), was launched at 1915 UT on November 3, 1994, (MSSTA II). Both launches occurred at the White Sands Missile Test Range in New Mexico under support of the NASA sounding-rocket experiment program. The MSSTA is a joint project of Stanford University, the NASA Marshall Space Flight Center (MSFC), and the Lawrence Livermore National Laboratory (LLNL). Its telescopes use multilayer coated normal-incidence optics in order to obtain greater collecting area and diffraction-limited resolution not offered by grazing incidence systems, $8,12,13$ and with spectral resolving powers, $\lambda / \Delta \lambda \sim 10-100$. These normal-incidence telescopes were tested prior to flight using standard interferometric techniques, and with high resolution test-targets at optical wavelengths. In-band wavelength point spread functions (PSF) can be inferred by the interferometric analysis of the optical performance data. Resolution tests were not performed at the telescopes' operating wavelengths because of the expense of operating at a laboratory such as the MSFC X-ray Calibration Facility which was especially designed for making this type of measurement. However, mirror and filter efficiency, and film responsivity measurements were carried out at the telescopes' operating wavelengths using synchrotron sources located at the Stanford Synchrotron Radiation Laboratory and the National Institute of Standards and Technology in Gaithersburg, Maryland.

At soft X-ray wavelengths, the MSSTA utilizes single reflection Herschelian optics to preserve as high a reflectivity as possible. For the longer wavelengths, the MSSTA employs doubly reflecting Ritchey-Chrétien and Cassegrain telescopes, which are compact designs allowing for longer focal lengths to achieve high resolution. The Cassegrain telescopes are not true Cassegrains in the sense that the optical elements were fabricated as spheres. Conic elements of the smoothness required for the multilayer coatings were unavailable at the time the Cassegrain optics were made. Small modifications were made to the payload instruments between the first and second flights; hence, we dub these flight payloads, MSSTA I and MSSTA II, respectively. The MSSTA I payload consisted of a total of 14 telescopes $(44 \AA-1550 \AA)$, as summarized in Table 1 below. MSSTA II had 19 telescopes covering the same wavelength range as MSSTA I; however, new telescopes were added or replaced with previous ones from the earlier flight (see Table 2). In all, MSSTA II carried six Ritchey-Chrétiens, two Cassegrains and eleven Herschelian telescopes. Another important difference between the payloads was the on-board electronics system which was completely redesigned in order to provide improved control of the in-flight operation of the experiment and provide real-time status information of the payload. This was relayed via a $1 \mathrm{Mbit} / \mathrm{sec}$ telemetry link to the ground station where we monitored the experiment with our "birdlink" program running on a Macintosh Quadra 840AV computer. The latter proved to be of major benefit in understanding the overall performance of the MSSTA payload, which we lacked during the 1991 flight. ${ }^{14-16}$ 
MSSTA I Telescopes Design Specifications (Flight 36.049)

\begin{tabular}{|c|c|c|c|c|c|}
\hline Telescope & $\begin{array}{c}\lambda \\
(\AA)\end{array}$ & Ion & $\begin{array}{c}\text { Solar atmosphere } \\
\text { temperature (K) }\end{array}$ & Focal length, $f(\mathrm{~mm})$ & f-ratio \\
\hline Ritchey-Chrétien - A & 1216 & $\mathrm{H} \mathrm{I}$ & $20,000-50,000$ & 3500 & 27.6 \\
\hline Ritchey-Chrétien - B & 1550 & C IV & 100,000 & 3500 & 27.6 \\
\hline Ritchey-Chrétien - C & 304 & $\mathrm{He} \mathrm{II}$ & 80,000 & 3500 & 27.6 \\
\hline Ritchey-Chrétien - D & 150 & $\mathrm{O} \mathrm{VI}$ & 300,000 & 3500 & 27.6 \\
\hline Ritchey-Chrétien - E & 173 & $\mathrm{Fe} I \mathrm{X} / \mathrm{X}$ & $600,000-1,000,000$ & 3500 & 27.6 \\
\hline Ritchey-Chrétien - F & 193 & Fe XII & $1,500,000$ & 3500 & 27.6 \\
\hline Ritchey-Chrétien - G & 335 & Fe XVI & $4,000,000$ & 3500 & 27.6 \\
\hline Cassegrain - A & 173 & $\mathrm{Fe} I X / \mathrm{X}$ & $600,000-1,000,000$ & 2000 & 33.2 \\
\hline Cassegrain - B & 211 & Fe XIV & $2,500,000$ & 2000 & 33.2 \\
\hline Herschelian - a & 143.3 & $\mathrm{Ne} \mathrm{V}$ & 400,000 & 1400 & 35.0 \\
\hline Herschelian - b & 44.1 & Si XII & $2,000,000$ & 1400 & 35.0 \\
\hline Herschelian - c & 44.1 & Si XII & $2,000,000$ & 1400 & 35.0 \\
\hline Herschelian - d & 132.8 & Fe XX & $8,000,000$ & 1400 & 35.0 \\
\hline Large Herschelian & 193 & Fe XII & $1,500,000$ & 1400 & 11.0 \\
\hline
\end{tabular}

Table 1. MSSTA I flight telescopes. The telescopes were designed and fabricated to transmit narrow bandpass radiation (spectral power resolution $\sim 10-100$ ) centered about the wavelength corresponding to emission produced by a particular ionic species in the Solar atmosphere. This allows one to image plasma regions of the Sun's atmosphere that define both differing morphology and physical processes corresponding to different temperatures.

\begin{tabular}{|c|c|c|c|c|c|}
\hline \multicolumn{6}{|c|}{ MSSTA II Telescopes Design Specifications (Flight 36.091) } \\
\hline Telescope & $\begin{array}{c}\lambda \\
(\boldsymbol{\AA}) \\
\end{array}$ & Ion & $\begin{array}{c}\text { Solar atmosphere } \\
\text { temperature }(\mathrm{K})\end{array}$ & Focal length, $f(\mathrm{~mm})$ & f-ratio \\
\hline Ritchey-Chrétien - I & 1216 & $\mathrm{HI}$ & $20,000-50,000$ & 2300 & 18.1 \\
\hline Ritchey-Chrétien - II & 193 & $\mathrm{Fe} \mathrm{XII}$ & $1,500,000$ & 3500 & 27.6 \\
\hline Ritchey-Chrétien - III & 150 & O VI & 300,000 & 3500 & 27.6 \\
\hline Ritchey-Chrétien - IV & 284 & $\mathrm{Fe} X \mathrm{XV}$ & $3,250,000$ & 3500 & 27.6 \\
\hline Ritchey-Chrétien - V & 304 & He II & 80,000 & 3500 & 27.6 \\
\hline Large Herschelian - VI & 93.9 & Fe XVII & $6,500,000$ & 1000 & 7.9 \\
\hline Ritchey-Chrétien - VII & 1550 & C IV & 100,000 & 3500 & 27.6 \\
\hline Cassegrain - 1 & 173 & $\mathrm{Fe} \mathrm{IX} / \mathrm{X}$ & $600,000-1,000,000$ & 2000 & 33.2 \\
\hline Cassegrain - 2 & 211 & Fe XIV & $2,500,000$ & 2000 & 33.2 \\
\hline Herschelian A & 211 & Fe XIV & $2,500,000$ & 1500 & 14.8 \\
\hline Herschelian B & 193 & Fe XII & $1,500,000$ & 1500 & 14.8 \\
\hline Herschelian - $\alpha$ & 44.1 & Si XII & $2,000,000$ & 1400 & 35.0 \\
\hline Herschelian - $\beta$ & 54.7 & Fe XVI & $4,000,000$ & 1000 & 25.0 \\
\hline Herschelian $-\gamma$ & 180 & $\mathrm{Fe} \mathrm{XI}$ & $1,250,000$ & 1250 & 32.8 \\
\hline Herschelian $-\dot{\delta}$ & 150 & O VI & 300,000 & 1000 & 25.0 \\
\hline Herschelian $-\varepsilon$ & 150 & O VI & 300,000 & 1000 & 25.0 \\
\hline Herschelian - $\zeta$ & 284 & $\mathrm{Fe} \mathrm{XV}$ & $3,250,000$ & 1200 & 30.0 \\
\hline Herschelian - $\eta$ & 54.7 & Fe XVI & $4,000,000$ & 1000 & 25.0 \\
\hline Herschelian - $\theta$ & 143 & $\mathrm{Ne} \mathrm{V}$ & 400,000 & 1400 & 35.0 \\
\hline
\end{tabular}

Table 2. MSSTA II: flight telescopes. This payload had 5 more telescopes than the first flight, but covered the same total wavelength range of $44-1550 \AA$.

In an earlier report we presented preliminary results of optical resolution measurements for the MSSTA II telescopes that had been made using photographic reproductions of a Standard 1951 Air Force High Resolution Test-target. 17 The present report is a follow-up to that analysis where we have re-analyzed those measurements, expanding our earlier study. Hence, we report on the same measured resolution tests of the MSSTA II payload telescopes. Design considerations and 
fabrication of the MSSTA instruments are discussed in previous publications by Walker et al. 18 and Hoover et al. 19 . For a complete description and report of the calibration measurements, i.e., optical characteristics, response functions and efficiency performance for all telescopes' mirrors, filters and photographic films from both flights we refer the reader to earlier studies by: Hoover et al., 20 Barbee et al., 21 Deforest et al., ${ }^{22}$ Allen et al., 23,24 Kankelborg et al., 25 and Plummer et al. 26 Recent solar physics analyses derived from both collections of MSSTA flight images can be found in recent publications by: Walker et al., 27, 28 Deforest et al., 29 Kankelborg et al., 30,31 Allen et al., 32 Oluseyi et al. 33 and Martínez-Galarce et al. 34

\section{MSSTA II - FLIGHT 36.091}

The Ritchey-Chrétien telescopes are the largest of the MSSTA payload. They are two-mirror telescopes with hyperboloid optical elements. The mirrors are held in place in their cells with a vacuum compatible Dow Corning Silastic RTV which also allows for dissipation of small motions introduced during rocket-launch vibrational loading of upto $\leq 18 \mathrm{~g}$ ' . To minimize changes in mirror separation, the optical benches were constructed of graphite fiber in an epoxy resin matrix, a material that has several desirable properties; most notably, strength, durability and light weight. ${ }^{19}$ Earlier theoretical design trade-studies by Hadaway et al. ${ }^{35}$ revealed that the Ritchey-Chrétien telescopes could obtain spatial resolutions of better than 0.3 arc-second over a 48 arc-min field of view at $1216 \AA$ (with careful control of all terms affecting resolution, a resolution of 0.1 arc-second appears as a reasonable goal; 36 further, we note that because of the short wavelength at which the MSSTA telescopes operate, diffraction is not the primary effect limiting resolution). The MSSTA II payload carried five Ritchey-Chrétien telescopes with mirrors coated to image bandpasses centered at 150, 193, 284, 304, and 1550A. A Short Ritchey-Chrétien telescope was also included in the payload and is capable of obtaining (diffraction-limited) resolutions of 0.24 arc-second at its operating wavelength of $1216 \AA .37$

The two Cassegrain telescopes (with coated optics designed to reflect bandpasses around 173 and $211 \AA$ ) were both flown on the MSSTA I payload. The $173 \AA$ telescope was flown on an earlier sounding-rocket flight payload, the StanfordMarshall Space Flight Center Rocket Spectroheliograph, launched on 1805 UT October 23, 1987. These Cassegrains are two-mirror telescopes with spherical optic elements, and when aligned and tested interferometrically, were found to be diffraction-limited at visible wavelengths. The maximum resolution, however, is limited to $\sim 0.5$ arc-second due to geometric aberrations. 1,11

The remaining eleven Herschelian telescopes flown were designed to operate at 44.1, 54.7, 93.9, 143, 150, 180, 193, 211, and $284 \AA$. All were off-axis paraboloids cut out of larger mastered mirrors of varying focal lengths; each mirror designed to reflect its respective off-axis component of the solar image. The $180 \AA$ telescope, which was a General Optics 1.5 inch concave sphere with a 2 meter radius of curvature was fabricated separately, and the multilayer coating for this optic was deposited at the MSFC by Palmer Peters a few weeks prior to launch. 38 All other substrate mirrors were fabricated by Baker Consulting and subsequently multilayer deposited at LLNL's Materials Science Laboratory by Troy Barbee, Jr., the exceptions being the Ritchey-Chrétien $1216 \AA$ and $1550 \AA$ mirrors which were coated by Acton Research Corporation. The FUV transmission filters were also manufactured by Acton Research while the EUV and XUV filters were fabricated by Forbes Powell of Luxel Corporation.

\subsection{Interferometric Focusing Measurements}

A few weeks prior to flight we tested the two Cassegrain and three of the six Ritchey-Chrétien telescopes interferometrically, for position of best focus, at the MSFC. The 284 and $1216 \AA$ telescopes were unavailable for testing during this period and the $1550 \AA$ telescope required special handling, as described later in this section. A Zygo PTI Fizeau interferometer, using a Helium-Neon laser $(6328 \AA)$ as its coherent light source, produced interferograms which were captured with a frame grabber (installed on an IBM-AT personal computer). These interferograms were then analyzed with the "MicroFringe 3.1" code. Because of the existence of the central obscuration, spider mount and multilayer sector boundaries on these telescopes, the interferogram contained many artifacts which the MicroFringe code was unable to recognize, and so the interference patterns were digitized manually. The entire set up was laid out on a $5^{\prime} \times 9^{\prime}$ Microflat $^{\prime}$ granite table. With a precision ( $1 / 20$ wave) flat reference mirror reflecting the beam back through the optical system under test, each fringe in the interferogram represents a $1 / 2$ wave of wavefront deformation. After moving the flat mirror to the point where MicroFringe 3.1 reported the flattest wave front, that position was then used as the position of best focus and, therefore, the camera film plane. The position of best focus for each telescope was found repeatably to within $0.025 \mathrm{~mm}$. A sample curve of the measured Modulation Transfer Function (MTF) for the $150 \AA$ Ritchey-Chretien telescope, as calculated from its interferogram, is shown in Figure 1. For the Ritchey-Chrétien (and Cassegrain) telescopes, a normalized spatial 
frequency of unity represents a diffraction-limited resolution of 1.25 arc-second (and 2.5 arc-second, at $6328 \AA$ ). Given these results, we expect the telescopes to be able to achieve the sub arc-second resolution at their respective operational wavelengths, based on diffraction-limited-only performance (when ignoring scattering effects, aberrations, source photon noise, pointing jitter, film noise, and thermal exposure). These calculations served as a base-line for the photographic tests later performed at the launch site just prior to flight and described in Section II.2.

The $1550 \AA$ Ritchey-Chrétien telescope was coated by Acton Research Corporation with a dielectric layer stack that has such a low reflectivity at the red He-Ne laser line that the multi-pass interferometer was unable to record an interference pattern and for this reason was not measured utilizing this technique. Hence, the conventional knife-edge test was performed instead. This test has a somewhat lower accuracy in determining the position of best focus; however the $1550 \AA$ RitcheyChrétien telescope operates at such a long wavelength that the depth of focus of the system is significantly greater than for the EUV and XUV instruments which can in part, compensate for some of the lost accuracy. 19 The remaining RitcheyChrétien telescopes could not be tested interferometrically and were not focused until just prior to flight at the launch site, due to time and financial constraints.

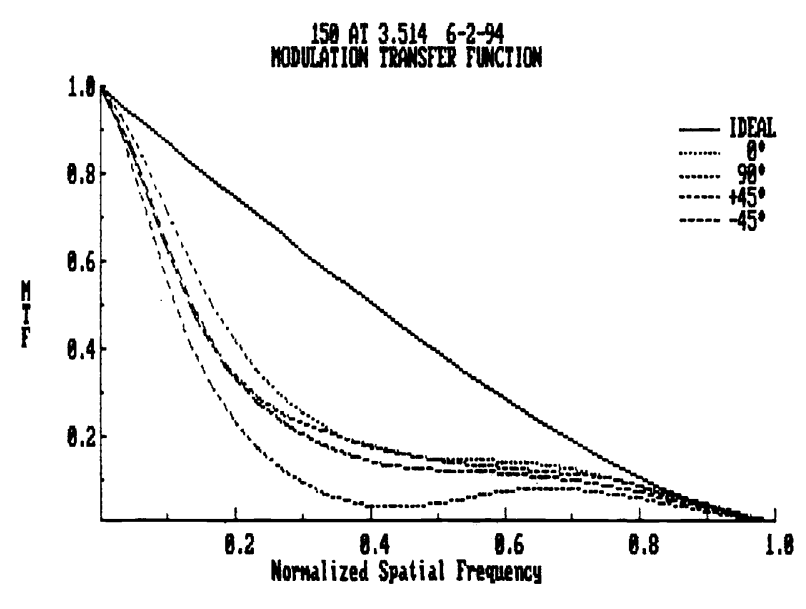

Figure 1. MTF Interferogram for the Ritchey-Chrétien $150 \AA$ Telescope. The abscissa measured frequencies are normalized to the Rayleigh Criterion for the Helium-Neon laser, $\lambda=6328 \AA$. Defocused phase shifts of $\pm 45^{\circ}$ and $90^{\circ}$ are shown. $0^{\circ}$ is best measured performance. Also shown are the theoretical ("Ideal") MTF curves for a system of equal aperture and focal length but with no central obscuration. Like determinations were made of the Ritchey-Chrétien 193 and $304 \AA$ telescopes, as well as the Cassegrain 173 and $211 \AA$ telescopes.

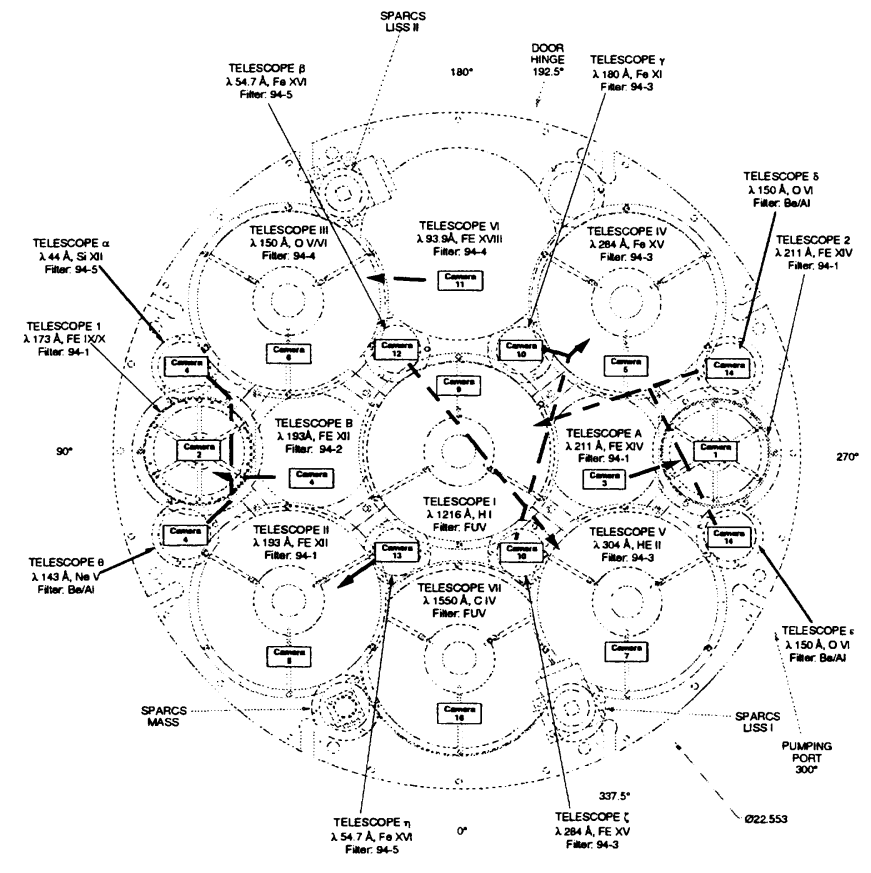

Figure 2. Configuration of MSSTA II telescopes as seen from a frontal view, looking into the apertures of all telescopes. In all, fourteen cameras, with fourteen metallic filters were setup to record 19 images (from 19 telescopes) onto $70 \mathrm{~mm}$ Kodak XUV-100 and 649 films.

\subsection{Pre-flight Alignment and Focusing}

All the telescopes were transported to the White Sands Missile Test Range, placed into the MSSTA II payload, and integrated with their cameras and electronics. Figure 2 depicts the positioning of each telescope as seen looking into the coaligned apertures from the front of the payload. Each telescope was aligned and focused onto a predesignated camera; with the exception of three cameras: \#4, \#10 and \#14, which had two or three telescopes focused onto their film planes. To aid in aligning their optical axes, a 16-inch Schmidt-Cassegrain collimator with a 180-inch focal length (manufactured by Diffraction Limited, Inc.) was used to illuminate the payload. This collimator was aligned and focused using a six inch cube's corner retro-reflector while a 10 arc-second pinhole was placed in its object plane. After one of the Ritchey-Chrétien telescopes was chosen as the "master" telescope and aligned in parallel to the central long axis of the payload, the collimator was aimed such that the pinhole was projected from the collimator and found exactly in the center of the "master" telescope's field of view. The remaining telescopes were subsequently aimed such that they too were imaging the pinhole in the center of their fields of view (for cameras \#4, \#10 and \#14 the pinhole was aligned elsewhere in the field of view due to the fact that 
these cameras were recording images from more than one off-axis Herschelian telescope on the same frame of film). This procedure aligned the telescopes to within one minute of arc.

To accurately point our payload toward the sun, Lockheed's Solar Pointing Attitude Rocket Control Systems (SPARCS) provided us with a Miniature Acquisition Sun Sensor (MASS) and two Lockheed Intermediate Sun Sensors (LISS). These sensors, combined, would send signals to the reaction control systems enabling our payload to acquire and track the sun to within 1 arc-second accuracy, and maintain pointing stability (jitter) to within $\leq 0.1$ arc-second. Because the LISS operates such that its entrance window is perpendicular to its optical axis, aligning it to our telescopes merely required centering the reflection of the pinhole from the sensor in the collimator while our "master" telescope also had the pinhole centered in its field of view. The MASS, however, required collimated sunlight to illuminate both types of sensors. When the LISS output a zero correction signal, the MASS was aligned by tilting it (using thin metal shims) until it also output a zero correction signal.

To perform 'in-the-field' focusing measurements, a Standard 1951 Air Force High Resolution Test-target was placed in the object plane of the collimator. A Gaertner traveling microscope, fitted with a razor blade in its image plane obscuring part of its field of view, was then used to view the image of the resolution test-target produced by each telescope. The position of best focus was chosen to be the location of the razor blade where the microscope showed the sharpest image. We were able to reproduce this position to within $\pm 1.02 \mathrm{~mm}$. After the cameras were mounted with their film planes in the correct position, photographs were taken of the resolution test-target at varying exposures. We present two sample test-target images in Figure 3 to illustrate the overall visible light focusing measurements of the telescopes.

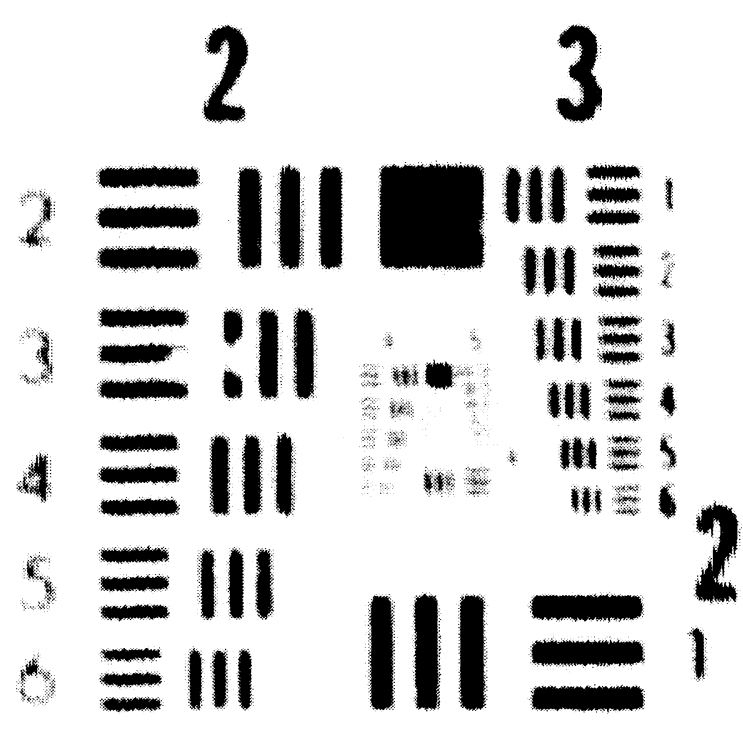

(a)

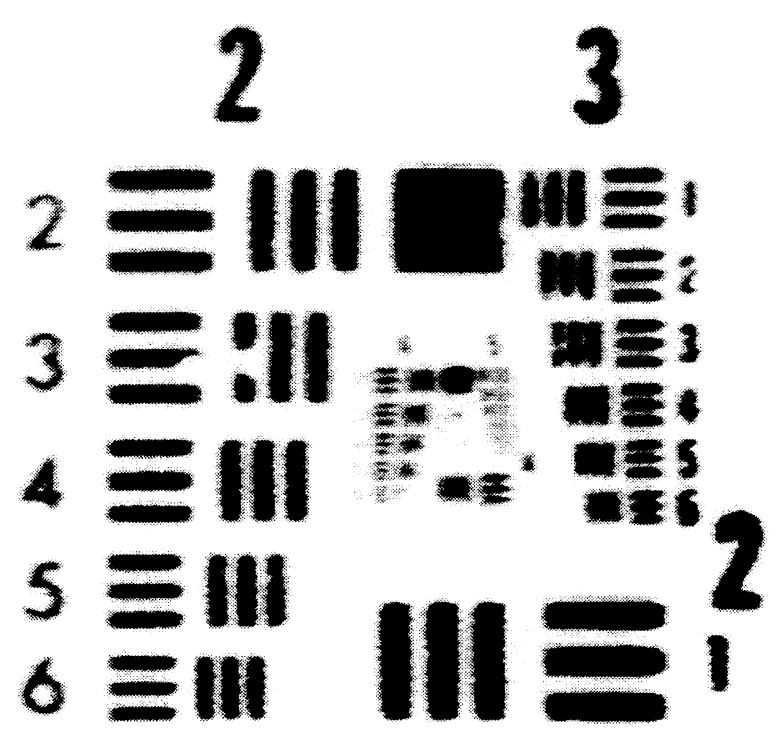

(b)

Figure 3: Visible focus test measurements using a standard 1951 Air Force High Resolution Test-target as reproduced by the (a) RitcheyChrétien $150 \AA$ and (b) Ritchey-Chrétien $1550 \AA$ telescopes. One can see the presence of jitter in both digital reproductions. In (a) the jitter was produced during the High Resolution Test-target measurements (possibly camera shutter or the facility's AC-system vibration), in (b) the effect is introduced by the stepper motor of the Ektron-Nikon Digitizer system.

Originally, due to the lower accuracy of this test, this method of focusing was only performed on the fourteen telescopes that were not or could not be interferometrically tested at the MSFC. After the payload had been vibration tested however, it was found that three of the secondary mirrors of the Ritchey-Chrétiens had moved (those of the $193 \AA, 284 \AA$ and $304 \AA$ telescopes), and so the mirrors were repositioned manually. These telescopes were then re-focused with the traveling microscope, as described previously. Due to the time constraint of the scheduled launch and limited resources, we were unable to devote more attention to carefully align and focus all telescopes to within diffraction-limited performance, as was discovered later during our resolution image analyses. 


\section{POST-FLIGHT TEST-TARGET RESOLUTION ANALYSES}

Our initial resolution performance results ${ }^{17}$ were based on viewing the resolution test-target images recorded on film under a microscope to determine which bar-sets in the horizontal and vertical positions were "resolvable". We were able to infer the corresponding angular resolution for each telescope based on a-priori knowledge of the bar-set geometries, as they are well defined and purportedly calibrated. Although this method is a sound approach in theory, it proved to be inaccurate due to some subjectivity and lack of superior measurement instrumentation. It also did not provide for a way to detect and quantify more accurately errors due to mirror tilt and misalignment, geometric aberrations, and film noise; which contribute to focusing errors. Hence, it proved to be a less rigorous approach, and we decided that a more accurate measurement was required. We determined therefore, that we had to measure the bar-set geometry (line spacings) directly and divide it by the telescope's focal length to obtain the telescope's true angular resolution as measured in visible light. In order to measure the film resolution target geometries, two methods of measurement were employed - the first, was by measuring carefully, calibrated, digitized reproductions of the resolution test-target images; and the second, was from direct line-space measurements of the bar-sets utilizing a line-width measuring microscope. Both approaches proved to be very accurate and reliable methods for measuring resolution performance.

\subsection{Digitization Measurement Procedure}

In order to digitally reproduce the images we needed to utilize an instrument capable of ultra-high-resolution at a spatial scale near $5 \mu$ on a pixel, and with at least 12-bits of $A / D$ dynamic range. The instrument that was chosen for our study is one that is located at the Image Processing Systems Laboratory of the Space Science and Astrophysics Division at the MSFC in Huntsville, Alabama. The instrument is an Ektron Model 1412 Digital Imaging Camera System, connected to a National Instruments NI-488 IEEE-488 Nubus Interface Card installed on an Apple Macintosh Quadra 950 computer. On. this computer we used version 3.0 of the Adobe Photoshop® software co-installed with the Ektron 1400-series Plug-in Module (v2.0) to monitor and control the Ektron digitizer and manipulate data output. All image data was saved in Tag Image File Format (TIFF) onto Compact Discs, and transferred to Stanford University for analysis.

The CCD device in the Ektron camera is a "scanning" linear array composed of 4096 pixels, each measuring (5 $\mathrm{x}$ 7) $\mu$ in size, with a $2 \mu$ pitch separation. (We say, "scanning" because it scans continuously within small steps using a stepper motor.) To take advantage of this spatial resolution we had to use a lens system on the camera that would allow accurate reproduction at a magnification scale of $1: 1$ or greater. We selected the Nikon $200 \mathrm{~mm}$ Micro-Nikkor $\mathrm{f} / 4 \mathrm{AF}$ (auto focus) lens, which provides 1:1 reproduction at a fixed minimum distance of $493.8 \mathrm{~mm}$ from object-to-image planes. Coupling the lens with a Nikon PK-13 Extension Ring allowed us increased magnification by effectively increasing the focal length of the lens system by $27.5 \mathrm{~mm}$ and imaging onto the CCD array behind the nominal focal plane. For this analysis, the use of extension rings proved unnecessary as the resolution capability of the Ektron Camera with the 200mm Nikon lens proved to be sufficient for most measurements, and in the cases where it did not we simply utilized a Nikon Measurescope (microscope) to measure test-target line-widths directly. Another reason we did not utilize the extension rings was that they intensified jitter effects detected during short integration time scans, that were due to the 'hanging' vertical positioning of lens and camera over the object plane. Hence, by adding the ring one effectively increases the length of the lens which translates to a larger pendulum swing for any vibration in the system, resulting in a magnified jitter effect seen in the digitized reproduction.

To calibrate and measure the resolution capability of the Ektron-Nikon digitizer system we also used a Standard 1951 Air Force High Resolution Test-target. This target was first measured (i.e., all line-width sets 0-1 through 7-6) using a Nikon Model II Measurescope connected to a Boecker Instruments MICROCODE II Digital Counter; which in turn had been calibrated using a standard line-width measuring target made by Graticules Ltd. of Kent, England. The repeatability of the Measurescope was found to be $\pm 0.1 \mu( \pm 3 \sigma)$. Figure 4 shows the Ektron-Nikon digitized image of the calibrated AF high resolution test-target. The measured resolution capability of the digitizer was observed down to line pairs 6-3 (horizontal) and 6-1 (vertical), corresponding to an average CCD pixel size of $6.34 \mu( \pm 0.25 \mu, \pm 3 \sigma)$ at $1: 1$ magnification.

\subsubsection{Diffraction and Measured Resolution}

Angular resolution, $\mathcal{R}$ is typically defined according to the standard Rayleigh Diffraction Criterion which places two "resolvable" objects at a well defined angular separation of

$$
\mathcal{R}=1.22 \frac{\lambda}{D} \equiv \mathcal{R}_{\text {Rayleigh }}(\lambda),
$$




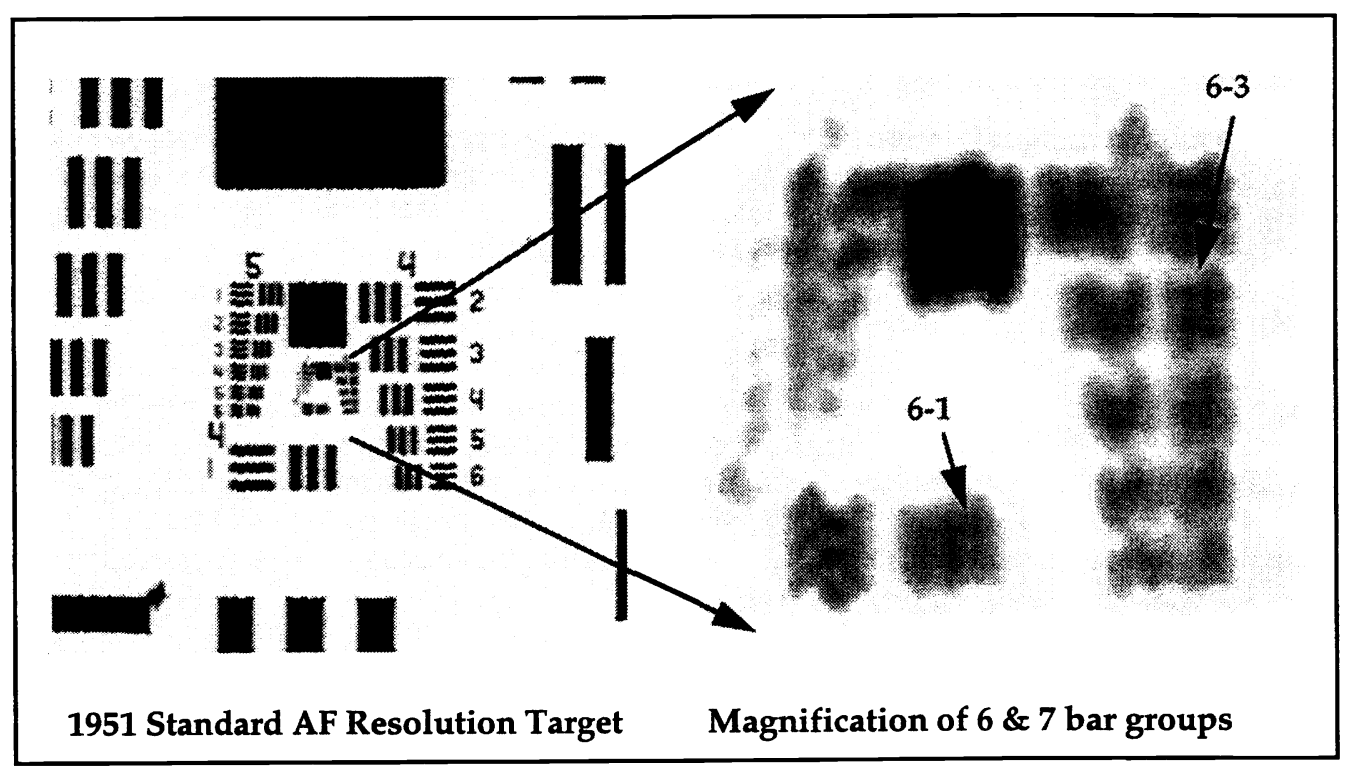

Figure 4. Left: digital image of Standard 1951 Air Force High Resolution Target taken with the Ektron-Nikon System. Right: $4 \mathrm{X}$ magnified view of 6 and 7 bar groups. Waviness of bar-sets is due to the vibration of the stepper-scanner motor in the digital camera and cooling fans of the light source, and is transferred to the focal plane and captured by the CCD. This vertical jitter corresponds to an error of $3 \sigma= \pm 1$ pixel in sinusoidal amplitude.

where $\lambda$ is the wavelength of light measuring the objects' separation imaged by a thin lens of aperture diameter, $D$. This definition is by no means an absolute measure of angular resolution but it is convenient as it places the center of one object's Airy disk onto the first zero of the adjoining object's Airy disk, thus providing a well-defined metric for "resolving" two objects at a given separation ${ }^{39}$ (Figure 5a). Given this definition however, it is conceivable that an optical system can outperform this metric as it is not necessary to utilize the Rayleigh Criterion for defining the resolvability of two objects. In the case of the Ritchey-Chrétien $150 \AA$ telescope for example, the diffraction-limit angular resolution, $R_{\text {Rayleigh }}(\lambda)=1.13$ arc-second, at $5700 \AA$, and the measured visible light values were found to be 0.70 and 0.94 arc-sec (for line pairs 5-4 and 53 , respectively).

The digitized reproductions of the AF high resolution test-target allows us to define resolvability of the MSSTA II telescopes by simply mapping the intensity profiles across two corresponding bar-sets in a test-target image that demonstrate the minimal "Rayleigh-like" curve characteristics, allowing us to measure the peak-to-peak separation of a resolution curve. This number is then divided by the focal length of the telescope which results in determination of the measured angular resolution of the telescope. In other words, the measured resolution, $\mathcal{R}_{m}$, is

$$
\mathcal{R}_{m}=\frac{\delta x}{f}
$$

where $\delta x$ is the peak-to-peak spatial separation of the resolution bar sets (in $\mu$ ), and $f$ is the telescope's focal length (in $\mu$ ) . This method was also utilized to determine the digitizer's resolution performance, as plotted in Figure 5(b). In Figure 6, we illustrate the intensity profiles for two of the Ritchey-Chrétien telescopes - the $150 \AA$ and the $1550 \AA$ instruments, and in Table 3 we present the visible resolution measurements for all MSSTA II telescopes.

We see from Table 3 that the measured performance for some telescopes are better than the predicted theoretical diffraction limit. In some cases the measurements are to within experimental error, but in those where it is not we can attribute the difference to the measurement not being exactly based on the definition of the Rayleigh Criterion as discussed previously (c.f. Eq. 1). We note from the criterion that two Airy peaks can be moved closer together and still be resolved, with no loss of generality to the theoretical definition (c.f. Figure 5 (a)). We believe this to be the case here as can be seen by the ratio of the valleys to peaks in the profiles of Figures 6 (a) and (b). The unevenness of the peaks in these figures is 
probably due to a combination of focus errors attributable to: jitter, geometric aberrations and optical misalignment; along with film, CCD, and quantization noise.

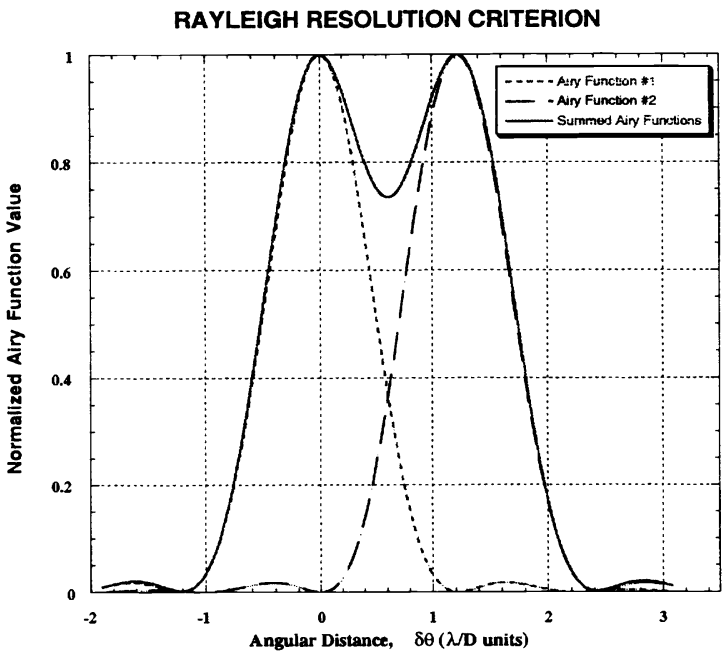

(a)

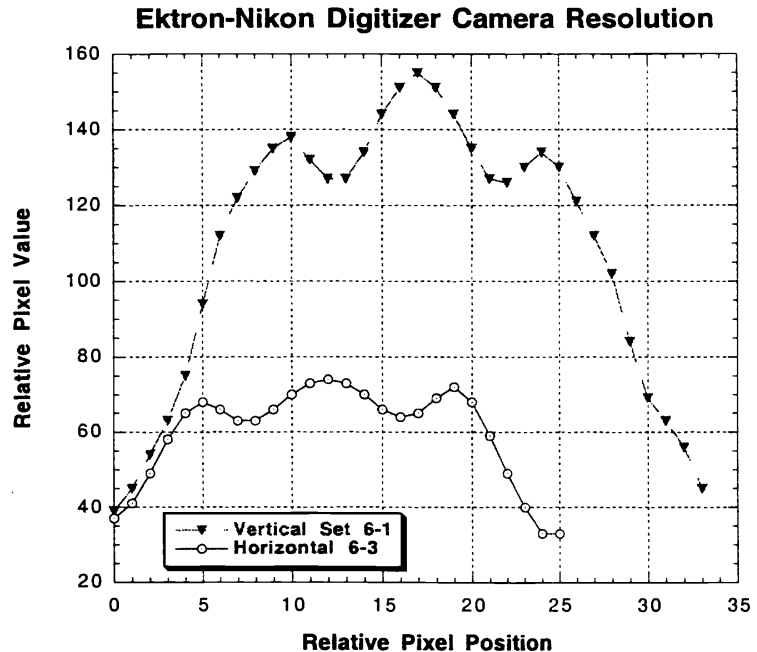

(b)

Figure 5. (a) Rayleigh Resolution Criterion: resolvability is measured as the separation of two adjoining Airy disks; where the maximum of one function coincides with the first zero of the adjacent curve, thus yielding an angular separation of $\delta \theta=1.22 \lambda / D$, where $\lambda$ is the wavelength of light; and $D$, the telescope aperture diameter. (b) Ektron 1412 Digital Camera Resolution Measurement of 1951 Standard Air Force High Resolution Target. Horizontal bar-set 6-3 and Vertical bar-set 6-1 are the smallest resolvable bar-sets which yield an average CCD pixel size of $6.34 \mu( \pm 0.25 \mu)$. Notice that the peak-to-peak separation of both bar-sets are equal.

\subsection{Microscope Line-Width Measurement Procedure}

The procedure to measure target resolution line-widths using the Nikon Measurescope is straight forward. The objective is to measure the peak-to-peak separation between lines in a barely resolvable bar-set on the film. Since the magnification of the Measurescope was sufficiently powerful to achieve this task it was unlikely that any large degree of subjectivity in the measurement played a role, and hence this error is relatively small; we estimate, no more than $\pm 2 \%$ accuracy. The principle error in measurement came from the repeatability error of the instrument. As reported above, this error was determined to be $\pm 0.1 \mu( \pm 3 \sigma)$. Although this proved to be the more accurate method of resolution measurement, it was not utilized for measuring all target film data. The chief purpose of using this instrument was to calibrate the high resolution test-target which in turn was used to calibrate the Ektron-Nikon digitizer system which we used for image digitization of all resolution target films, and, MSSTA I and MSSTA II solar spectroheliograms. This was a necessary procedure as it is critical for us to have a solid understanding of the errors involved in the digitization process, as well as to have a well calibrated measure of the digital pixel size, and an estimate of the digitizer's PSF.

Initial measurements reported by Gore et al. ${ }^{17}$ proved to be about a factor 2 higher in some cases than the current figures in Table 3. This discrepancy is due to the fact that the resolution target films for each telescope were never measured directly with a line-width measuring microscope as was done in this study. Also, the magnification of the microscope used in the earlier measurements proved to be insufficient and gave a strong level of subjectivity to interpretation - due to human observation of which line-pairs looked resolved, coupled with simple optics geometry considerations. This technique led to inaccurate conclusions and did not give us a clear understanding of what possible focusing errors were present. The current analysis however, provides a method to quantify these measurements and demonstrate results that meet the most important goal of the MSSTA mission - i.e., to obtain experimentally, the highest angular resolution spectroheliograms in FUV, EUV and soft X-ray wavelengths. 
Ritchey-Chrétien 150Å Telescope Resolution

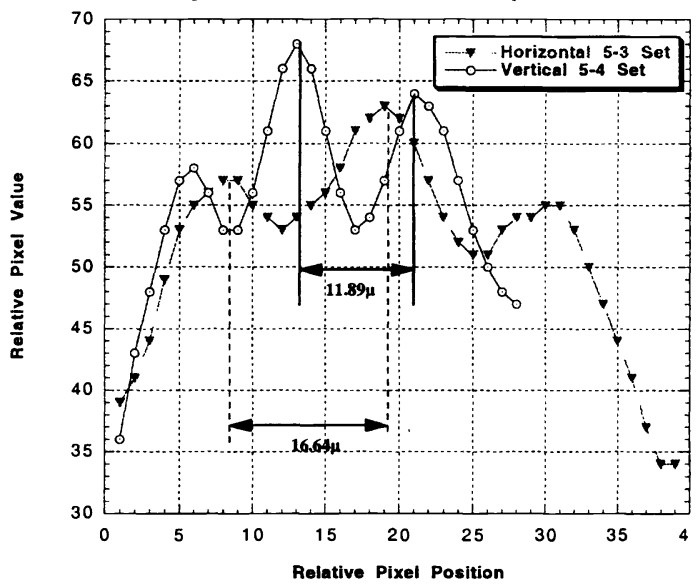

(a)

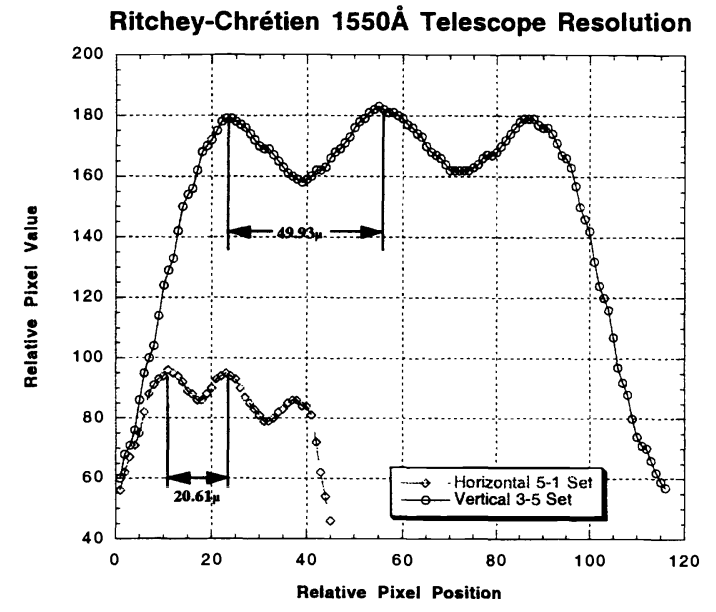

(b)

Figure 6. (a) Ritchey-Chrétien $150 \AA$ Telescope resolution measurement at 0.94 " in horizontal and 0.70 " in vertical directions. (b) RitcheyChrétien $1550 \AA$ Telescope resolution measurement at 1.21 " in horizontal and 2.94 " in vertical directions. The focal length of the RitcheyChrétien telescopes is $3500 \mathrm{~mm}$. Values shown in plots are real spacial separations between peaks as measured on the film.

\section{IMAGE DIGITIZATION MODEL}

The final step in the analysis was to insure that the above measuring technique was correct, and to understand in detail the digitization process. Hence, we modeled the procedure that produces a digitized image, beginning from the point where an image enters the telescope aperture to the point when it is recorded on the digital detector (Ektron CCD camera). In Figure 7 we depict this process schematically. The model we determined accurately predicts the physical digitization process of a resolution test-target image (or solar spectroheliogram image) from beginning to end.

In the first step of Figure 7, $f(x, y)$, the resolution test-target image (or solar image) plane wave, enters the telescope aperture. It then convolves with the point-spread function (PSF) of the telescope, $h(x, y)$, to generate the image, $g(x, y)$, before it strikes the film. However, in order to account for errors such as payload jitter, film noise, scatter, and photon Shot noise, we add gaussian noise, $n_{f}(x, y)$, to the image to produce $k(x, y)$, the image that is actually recorded onto the film. The next step involves the digitization of the film image. Here the film image is first convolved with a sampling function (or Shah function, $\left.{ }^{2} I I(x, y)\right){ }^{40}, S\left(x-x_{i}, y-y_{j}\right)$, which produces a sampled image, $I\left(x_{i}, y_{j}\right)$; that depicts accurately the quantization of an image by a CCD. Finally, to account for CCD noise (electronic Shot noise, thermal noise, $1 / f$ noise, etc. ${ }^{41}$ ) and jitter due to the scanner stepper motor and the cooling fans of the light source sitting below the film sample, we add noise, $n_{d}\left(x_{i}, y_{j}\right)$, to produce the digitized image, $\hat{f}\left(x_{i}, y_{j}\right)$, that is written as 8-bit (or 16-bit) TIFF data. In applying this model to the resolution test-target patterns we see in Figure 8 that indeed we obtain results that are comparable to what we determined above when measuring the intensity profiles across barely-resolvable test-target bar-sets (Figure 6). This lends confidence to our interpretation of the test-target profile measurements.

We note that actual film digitization of a spectroheliogram involves two PSF convolution steps - the first is the object image convolved with a MSSTA telescope and the second is the film image convolved with the Ektron-Nikon digitizer camera. Since we make the simplifying assumption that the system response is spatially invariant, or equivalently, isoplanatic - this allows us to model the digitization process as a single PSF convolution; which is true for linear imaging systems. Hence, we are able to incorporate the PSF response of both the MSSTA telescopes and the digitizer camera as a single PSF response in our model. Although not measured, we know from earlier theoretical analyses that the MSSTA Ritchey-Chrétien telescopes' system response are linear; hence, as the PSF is isoplanatic over a field of view of $\sim 48$ arcminutes 35 our image model is viable. Spatial invariance was also observed over $85 \%$ of the total field-of-view of the Ektron-Nikon Digitizer system via flat-field digitization measurements. However, because we do not have true knowledge of 
each telescopes' operating wavelength PSF we can only estimate it using the current estimates provided in this analysis coupled with semi blind-iterative deconvolution techniques $42-45$ in order to deconvolve the digitized solar images. In the present analysis we do not discuss the application of these techniques as we intend to report these findings in a separate study at a later date.

MSSTA II Telescopes Visible Light Resolution Performance Measurements

\begin{tabular}{|c|c|c|c|c|c|}
\hline Telescope & $\begin{array}{c}\lambda \\
(\AA)\end{array}$ & $\begin{array}{c}\text { Resolution Target } \\
\text { Bar-set } \\
\text { Resolved } \\
\end{array}$ & $\begin{array}{c}\text { Measured } \\
\text { Resolution } \mathcal{R}_{n,} \\
\text { (arc-second) }\end{array}$ & $\begin{array}{c}\text { Measurement } \\
\text { Error }( \pm 1 \sigma, \text { arc-second })\end{array}$ & $\begin{array}{l}\text { Diffraction Limit } R_{\text {rayleigh }} \\
\text { (@ } \lambda 5700 \AA \text { - arc-second) }\end{array}$ \\
\hline Ritchey-Chrétien - I & 1216 & $\begin{array}{l}\text { Horiz. 5-3 } \\
\text { Vert. 4-4 }\end{array}$ & $\begin{array}{l}1.08 \\
1.79\end{array}$ & $\begin{array}{l}0.09 \\
0.10\end{array}$ & 1.13 \\
\hline Ritchey-Chrétien - II & 193 & $\begin{array}{c}\text { Horiz. 4-5 } \\
\text { Vert. 4-5 }\end{array}$ & $\begin{array}{l}1.06 \\
1.37\end{array}$ & $\begin{array}{l}0.11 \\
0.09\end{array}$ & 1.13 \\
\hline Ritchey-Chrétien - III & 150 & $\begin{array}{l}\text { Horiz. 5-3 } \\
\text { Vert. 5-4 }\end{array}$ & $\begin{array}{l}0.94 \\
0.70\end{array}$ & $\begin{array}{l}0.06 \\
0.08\end{array}$ & 1.13 \\
\hline Ritchey-Chrétien - IV & 284 & $\begin{array}{l}\text { Horiz. 5-3 } \\
\text { Vert. 3-4 }\end{array}$ & $\begin{array}{l}1.24 \\
3.42\end{array}$ & $\begin{array}{l}0.06 \\
0.41\end{array}$ & 1.13 \\
\hline Ritchey-Chrétien - V & 304 & $\begin{array}{l}\text { Horiz. 5-3 } \\
\text { Vert. 5-3 }\end{array}$ & $\begin{array}{l}1.12 \\
1.12\end{array}$ & $*$ & 1.13 \\
\hline Large Herschelian - VI & 93.9 & $\begin{array}{l}\text { Horiz. 4-5 } \\
\text { Vert. 2-6 }\end{array}$ & $\begin{array}{l}1.65 \\
5.98\end{array}$ & $\begin{array}{l}0.21 \\
0.24\end{array}$ & 1.13 \\
\hline Ritchey-Chrétien - VII & 1550 & $\begin{array}{l}\text { Horiz. 5-1 } \\
\text { Vert. 3-5 }\end{array}$ & $\begin{array}{l}1.21 \\
2.94\end{array}$ & $\begin{array}{l}0.12 \\
0.08\end{array}$ & 1.13 \\
\hline Cassegrain - 1 & 173 & $\begin{array}{l}\text { Horiz. 3-3 } \\
\text { Vert. 2-6 }\end{array}$ & $\begin{array}{l}4.03 \\
5.98\end{array}$ & $\begin{array}{l}0.36 \\
0.16\end{array}$ & 2.38 \\
\hline Cassegrain - 2 & 211 & $\begin{array}{l}\text { Horiz. 3-5 } \\
\text { Vert. 3-1 }\end{array}$ & $\begin{array}{l}2.07 \\
3.38\end{array}$ & $\begin{array}{l}0.19 \\
0.19\end{array}$ & 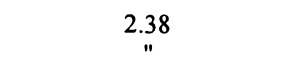 \\
\hline Herschelian A & 211 & $\begin{array}{l}\text { Horiz. 4-5 } \\
\text { Vert. 3-5 }\end{array}$ & $\begin{array}{l}1.38 \\
2.58\end{array}$ & $\begin{array}{l}0.14 \\
0.12\end{array}$ & 1.20 \\
\hline Herschelian B & 193 & $\begin{array}{l}\text { Horiz. 3-4 } \\
\text { Vert. 3-3 }\end{array}$ & $\begin{array}{l}3.20 \\
4.36\end{array}$ & $\begin{array}{l}0.36 \\
0.21\end{array}$ & 1.20 \\
\hline Herschelian $-\alpha$ & 44.1 & $\begin{array}{l}\text { Horiz. 3-4 } \\
\text { Vert. 3-2 }\end{array}$ & $\begin{array}{l}3.39 \\
4.57\end{array}$ & $\begin{array}{l}0.16 \\
0.17\end{array}$ & 3.59 \\
\hline Herschelian - $\beta$ & 54.7 & $\begin{array}{l}\text { Horiz. 0-6 } \\
\text { Vert. 0-3 }\end{array}$ & $\begin{array}{l}26.81 \\
30.94\end{array}$ & $\begin{array}{l}0.57 \\
0.65\end{array}$ & 3.59 \\
\hline Herschelian $-\gamma$ & 180 & $\begin{array}{l}\text { Horiz. 0-5 } \\
\text { Vert. 0-6 }\end{array}$ & $\begin{array}{l}21.45 \\
17.26\end{array}$ & $\begin{array}{l}1.57 \\
1.74\end{array}$ & 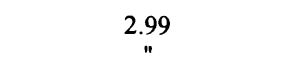 \\
\hline Herschelian $-\delta$ & 150 & $\begin{array}{l}\text { Horiz. 2-6 } \\
\text { Vert. 3-4 }\end{array}$ & $\begin{array}{l}5.45 \\
3.92\end{array}$ & $\begin{array}{l}0.38 \\
0.22\end{array}$ & $\begin{array}{c}3.59 \\
" ~\end{array}$ \\
\hline Herschelian $-\varepsilon$ & 150 & $\begin{array}{c}\text { Horiz. 1-5 } \\
\text { Vert. 2-2 }\end{array}$ & $\begin{array}{l}14.23 \\
8.50\end{array}$ & $\begin{array}{l}0.35 \\
1.13\end{array}$ & 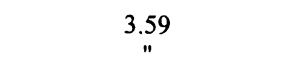 \\
\hline Herschelian $-\zeta$ & 284 & $\begin{array}{c}\text { Horiz. 2-6 } \\
\text { Vert. 2-1 }\end{array}$ & $\begin{array}{l}4.09 \\
7.63\end{array}$ & $\begin{array}{l}0.34 \\
0.39\end{array}$ & 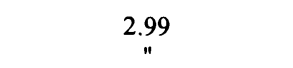 \\
\hline Herschelian - $\eta$ & 54.7 & $\begin{array}{c}\text { Horiz. 3-5 } \\
\text { Vert. 3-4 }\end{array}$ & $\begin{array}{l}3.09 \\
3.51\end{array}$ & $\begin{array}{l}0.22 \\
0.22\end{array}$ & 1.79 \\
\hline Herschelian - $\theta$ & 143 & $\begin{array}{c}\text { Horiz. 3-4 } \\
\text { Vert. 2-4 }\end{array}$ & $\begin{array}{l}3.39 \\
6.54\end{array}$ & $\begin{array}{l}0.16 \\
0.38\end{array}$ & $\begin{array}{c}1.79 \\
"\end{array}$ \\
\hline
\end{tabular}

Table 3. Visible light resolution test measurements of the MSSTA II telescopes from photographic reproductions of a 1951 Standard Air Force High Resolution Test-target. Theoretical diffraction limits are calculated using $\lambda=5700 \AA$. *Records show that the RitcheyChrétien $304 \AA$ telescope was indeed aligned and focused at White Sands, but at the time of this analysis, we were unable to locate the resolution test-target film images taken with this telescope and hence, have no measured error for this instrument.

We have observed that in some cases the PSF of a MSSTA telescope was greater than the PSF of the digitizer and hence dominate the focusing error of the digitized image reproduction. One such comparison is demonstrated in Figures 9 and 10 with digitizations of two of the spectroheliograms recorded simultaneously during the MSSTA II flight. Figure 9 is that of the Ritchey-Chrétien $1550 \AA$ telescope (C IV ion plasma at $\sim 10^{5} \mathrm{~K}$ ), and Figure 10 is that of the small Herschelian telescope operating at $284 \AA$ (Fe XV ion plasma at $\sim 3.2 \times 10^{6} \mathrm{~K}$ ). Both were recorded on Kodak 649 film which has a line resolution of 2000 lines $/ \mathrm{mm}$. It can be seen that the clarity of detail is much sharper in the $\mathrm{C} \mathrm{IV} \mathrm{image} \mathrm{while} \mathrm{the} \mathrm{Fe} \mathrm{XV}$ image is blurry and is slightly out of focus. From the above resolution measurements (Table 3 ) we determined that the Ritchey-Chrétien $1550 \AA$ telescope performed partially within its visible wavelength diffraction limit, while the Herschelian 


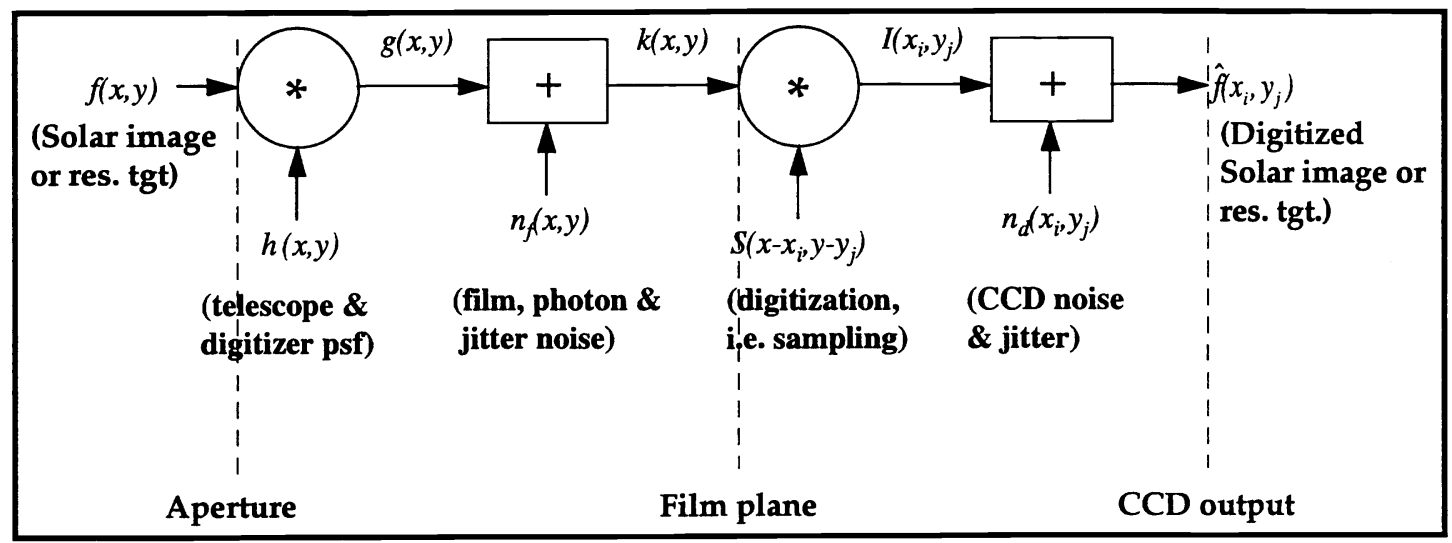

Figure 7. Digitization Model of object image through recorded digitization. Note that this model incorporates the telescope PSF and digitizer camera PSF as a single-step convolution with the source image, a natural consequence of the Fourier transform property of the Convolution Theorem for an isoplanatic PSF.

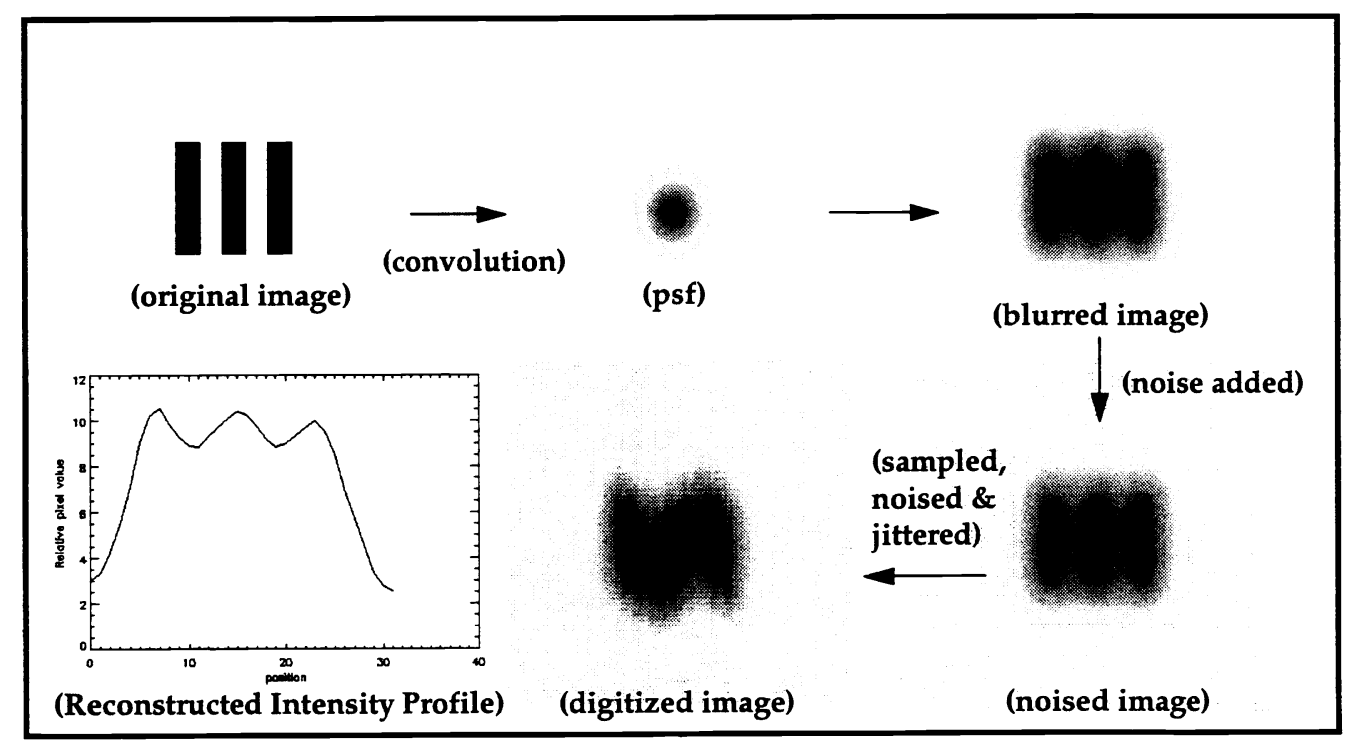

Figure 8: The digitization model begins from the top-left corner with a well-defined bar-set. It is then convolved with a slightly elongated PSF (parallel in direction to the bars' length), noised (additively), then down-sampled, jittered and noised again (additively). Finally, from this down-sampled, noised image, we take the intensity profile across the width of the bar-set and obtain the expected result seen in the lower left-hand corner.

$284 \AA$ was roughly a factor of 2 greater than its expected visible wavelength performance. Because the visible light measurements for the Ritchey-Chrétien $1550 \AA$ telescope were near the visible diffraction limit, we estimate that the focus errors were negligible compared to the diffraction limit and hence that this telescope achieved sub arc-second resolution near its theoretically designed operating FUV wavelength of 0.31 arc-second (not including pointing jitter or film resolution). We conclude therefore, that the evidence of the observed visible light resolution measurements indicate that:

the performance of certain MSSTA II telescopes, at or near, their diffraction limit in visible wavelengths shows that any figure of focus errors were small compared to the visible light diffraction limit. Based on these measurements, we estimate that these telescopes performed at sub arc-second resolution at their respective operating bandpass. 


\section{OPERATING WAVEBAND RESOLUTION PERFORMANCE}

\subsection{Thin Lens Optical Systems}

In order to support the latter hypothesis we consider the following argument: we know that the Rayleigh Criterion (Section 3.1) is a consequence of classical electromagnetism and the Fourier properties of thin lenses. In the thin lens approximation the PSF is the Fourier transform of a plane wave that emanates from a point source placed at a distance satisfying the Fraunhofer diffraction condition. ${ }^{39}$ The system PSF directly measures the highest spatial frequency response of the optics and, because the lens diameter $D$ is finite, reproduction of all spatial frequencies embedded in the point source is also finite resulting in limited image reproduction. Therefore, in order to completely reproduce a point source we would require a lens of infinite diameter to render the angular resolution of the PSF, as given by Eq. (1), to equal zero as would be expected with a point source. However, because of the quantum nature of light and matter this definition for optical resolution is only partially correct as it accounts only for an ideal classical, diffraction-limited, linear optical system. In practice one must take other effects into account, such as: aberrations, pointing jitter, transmission screen (filter) variations, detector noise, photon noise and scattering for example - all of which alter the functional form of the system PSF over the image plane.

A photon, as it traverses through an optical system, will interact with each physical element (a mirror, lens, filter, detector, etc.) in such a way as to sufficiently alter its physical properties to produce a result that can be described by a series of independent random probability distributions. Because these interactions are often independent and uncorrelated to one another the end result must be the superposition of these independent events (each described by a probability distribution) and is therefore random. In the limit where the frequency of occurrence of a random process, $N$, is large $(N \rightarrow \infty)$, the resulting probability density function will tend toward a normal (Gaussian) distribution. Equivalently, the sum of a series of coincident probability densities will also tend toward a normal density distribution as $N \rightarrow \infty$. This result is the well known consequence of the Central Limit Theorem of statistics. 46 Hence, as $N$ independent photons travel through an optical system, each physical interaction with the photon will produce a probability density distribution describing that interaction, with the resulting measured process behaving as a Gaussian random process of size $N$.

\subsection{Linear Combination of Normal (LCN) Random Variables}

If we consider $n$ independent random variables, $\left\{X_{1}, X_{2}, \ldots, X_{n}\right\}$, with means $\left\{\mu_{1}, \mu_{2}, \ldots, \mu_{n}\right\}$ and variances $\left\{\sigma_{1}^{2}, \sigma_{2}^{2}, \ldots \sigma_{n}^{2}\right\}$; and if we denote $Y$ as the random variable that is a linear combination of the $X_{i}$ 's given by

$$
Y=a_{1} \mathrm{X}_{1}+a_{2} \mathrm{X}_{2}+\ldots+a_{n} \mathrm{X}_{n}
$$

where the $a_{i}$ 's are constants; then the expectation value, $E(Y)$, we know is given by

$$
E(Y)=\mu_{y}=a_{1} \mu_{1}+a_{2} \mu_{2}+\ldots+a_{n} \mu_{n} .
$$

Similarly, the expected variance is found to be

$$
\begin{aligned}
E\left[\left(Y-\mu_{Y}\right)^{2}\right] & =\sigma_{Y}^{2} \\
& =a_{1}^{2} \sigma_{1}^{2}+a_{2}^{2} \sigma_{2}^{2}+\ldots+a_{n}^{2} \sigma_{n}^{2} .
\end{aligned}
$$

The consequence of the above is simply that if the $X_{i}$ s are normally distributed random variables, then so will $Y$ be normally distributed with mean $\mu_{Y}$ and variance $\sigma_{Y}^{2}$. Eq (5) indicates that the expected variance of random variable $Y$ is the sum of the expected variances for the individual random variable $X_{i}$ distributions. 


\subsection{Image Resolution Error Budget}

For an imaging optical system the resulting PSF can be approximated by a Gaussian distribution. This is true even in the limit where the system is purely diffraction limited with no focus figure errors - as the central lobe of the Airy function can be approximated by a 2-dimensional normal distribution. One standard measure of the width of a normal distribution is given by the full-width-at-half-maximum (FWHM). The FWHM is related to the standard deviation, $\sigma$, by

$$
F W H M=2.35 \sigma .
$$

In an optical system where the errors that affect focus are physically uncoupled and statistically uncorrelated to one another, without loss of generality, the $a_{i}$ 's in equations (3) - (5) can be set equal to one (as well as be absorbed into their respective $\sigma_{i}$ factor). This reduces eq (5) to the sum

$$
\sigma_{Y}^{2}=\sigma_{1}^{2}+\sigma_{2}^{2}+\ldots+\sigma_{n}^{2} .
$$

Substituting eq (6) into (7), simply yields

$$
F W H M_{Y}^{2}=F W H M_{X_{1}}^{2}+F W H M_{X_{2}}^{2}+\ldots+F W H M_{X_{n}}^{2} .
$$

The latter equation therefore states that the resulting $F W H M_{Y}$ is the square root of the quadrature sum of all $F W H M$ for independent random variable distributions, $X_{i}$. Interpreted in another manner, this states that for an optical system the resolution error budget is summed in quadrature for errors that are independent and uncorrelated, or equivalently, form a LCN random variables. Hence, at a given wavelength $\lambda$ and radial field position $r$ relative to the optical axis, the measured resolution, $\mathcal{R}_{m}(r, \lambda)$, for a telescope operating near its diffraction limit can be defined as the $F W H M$, given by

$$
\mathcal{R}_{m}^{2}(r, \lambda)=\mathcal{R}_{\text {Rayleigh }}^{2}(r, \lambda)+\varepsilon_{\theta}^{2}(r, \lambda)
$$

where $\mathcal{R}_{\text {Rayleigh }}(r, \lambda)$ is the expected diffraction-limited resolution (i.e. Rayleigh Criterion), and $\varepsilon_{\theta}(r, \lambda)$ is defined as the resolution focusing error which is the square-root-quadrature sum of all statistically uncorrelated errors, $\delta_{\theta_{i}}(r, \lambda)$, affecting resolution at wavelength $\lambda$. These errors can include: geometrical aberrations, transmission screen distortion, mirror tilt, optic misalignments, photon noise, source motion, pointing jitter and detector noise. In other words, $\varepsilon_{\theta}(r, \lambda)$, is

$$
\varepsilon_{\theta}(r, \lambda)=\sqrt{\sum_{i=1}^{n} \delta_{\theta_{i}}^{2}(r, \lambda)}=\sqrt{\delta_{\theta_{1}}^{2}(r, \lambda)+\delta_{\theta_{2}}^{2}(r, \lambda)+\ldots+\delta_{\theta_{n}}^{2}(r, \lambda)}
$$

where the subscript $\theta_{i}$ refers to each of the $n$ uncorrelated random errors that contribute to the net resolution. Eq. (10) therefore, shows the error budget distribution of uncorrelated focusing errors in an optical system.

In a previous paper Walker et al. ${ }^{36}$ performed a theoretical trade study of the expected error budget for the MSSTA telescopes and found that the geometric aberrations and diffraction dominate the net resolution. In the present work we were unable to experimentally measure each of the optical system errors directly; however, we estimate an upper limit to the total resolution focusing error, $\varepsilon_{\theta}(r, \lambda)$, which comprises the root-sum-square of all errors affecting resolution. As described previously, the MSSTA II telescopes were aligned and focused on the ground at visible wavelengths just prior to launch. These measurements, $\mathcal{R}_{m}\left(r, \lambda_{\text {visible }}\right)$, listed in Table 3, are described by Eq. (9). If, for every telescope, we subtract the corresponding square of the visible diffraction limit, $R_{\text {Rayleigh }}\left(r, \lambda_{\text {visible }}\right)$, from the square of each measured value, $\mathcal{R}_{m}\left(r, \lambda_{\text {visible }}\right)$; then the square root of this difference yields an upper limit estimate of the resolution focusing error, $\varepsilon_{\theta}\left(r, \lambda_{\text {visible }}\right)$, for each telescope as measured in visible wavelengths. In other words, 


$$
0 \leq \varepsilon_{\theta}\left(r, \lambda_{\text {visible }}\right)=\sqrt{\mathcal{R}_{m}^{2}\left(r, \lambda_{\text {visible }}\right)-\mathcal{R}_{\text {Rayleigh }}^{2}\left(r, \lambda_{\text {visible }}\right)}
$$

We assert that this error is an upper-bound estimate of the MSSTA II telescopes' resolution focus figure errors that largely include: geometric aberrations, mirror tilt and misalignments, and geometrical effects from the mismatch of off-axis mirror segments to their (originally) assigned film planes. (Such errors are optical system errors and are in fact, wavelength independent.) The latter effects were the dominant errors attributable to the large focusing errors measured for 13 of the 19 telescopes which did not achieve diffraction-limited performance. At visible wavelengths, these errors are significantly larger than those caused by scattering effects due to mirror surface imperfections and roughness or pointing jitter present during the visible light resolution tests. Further, the difference in measured resolution of orthogonal test-target line pairs was a consequence of mirror tilt and misalignment caused either during transport of the payload to the White Sands Missile Test Range facility or during pre-flight vibration testing which resulted in our having to manually re-align and re-focus three of the Ritchey-Chrétien telescopes without the aid of interferometric precision alignment.

\subsection{Effect of Multilayer Surface Imperfections on Resolution Performance}

Zmek et al. 47 and Harvey et al. 48 have modeled the impact of minor surface imperfections on image quality for multilayer mirrors operating at soft X-ray and EUV wavelengths. The power spectral density (PSD) of minor surface imperfections, $\sigma^{2}\left(f_{x}, f_{y}\right)$, may be expanded as: 12

$$
\sigma^{2}\left(f_{x}, f_{y}\right)=\left(\frac{1}{A}\right)\left|\iint_{A} z(x, y) \exp \left[2 \pi i\left(f_{x} x+f_{y} y\right)\right] d x d y\right|^{2}
$$

where $z(x, y)$ represents the deviation of the real mirror from the surface of an ideal mirror at point $(x, y) ;\left(f_{x}, f_{y}\right)$ represent spatial frequencies in Fourier space; and the integral is taken over the area, $A$, of the mirror. The quantity $\sigma^{2}\left(f_{x}, f_{y}\right)$ may be measured over specific frequency ranges using various techniques, as described by Spiller et al. ${ }^{12}$, to provide an average surface roughness over some range of surface frequencies, $\Delta f$,

$$
\sigma_{\Delta f}^{2}=2 \pi \int_{\Delta f} \sigma^{2}(f) f d f
$$

Harvey et al. 48 divide the surface roughness into three spatial frequency domains, low frequency ( $\sigma_{L}$, on the scale on millimeters or larger); high frequency microroughness, ( $\sigma_{H}$ on the scale of 0.01 microns or less); and mid-frequency roughness $\left(\sigma_{M}\right)$. Microroughness degrades multilayer efficiency. However, because scattering at the various multilayer interfaces is uncorrelated, scattering by imperfection on this scale is attenuated by factor $1 / \sqrt{L}$, where $L$ is the number of reflecting layers. The very high reflectivity at their respective working wavelengths indicates that this effect is small for our mirrors. Interferometric tests performed in earlier analyses by Hoover et al. ${ }^{19}$ demonstrate that figure errors are also small for the MSSTA mirrors. Control of mid-frequency surface roughness, then, becomes critical for the performance of normalincidence multilayer mirrors. Both Harvey et al. ${ }^{48}$ and Spiller et al. 12 evaluate its effects. It is difficult to measure midfrequency surface roughness density as it is generally assumed that surface roughness scales as, $b f^{-\alpha}$, where $b$ is determined from surface microroughness measurements, and $2 \leq \alpha \leq 3$. The microroughness of our mirrors, $\sigma_{H} \sim 1-2 \AA$ RMS, is $\sim 3$ times lower than the values selected by Harvey et al. in their study; and, based on their analysis $\sim 85 \%$ of the energy from a point source will be within a circle of diameter 0.06 arc-second at $304 \AA$ for a mirror with $\sigma_{H} \sim 5 \AA$ RMS. The analysis of Spiller et al. predicts that for their Herschelian mirror, which operates at $\sim 63 \AA$, the measured spectral density of defects should produce observable effects in their images. Since such effects were not observed, they conclude that their 
measurements represent an upper limit to the actual roughness at mid-frequencies. Because the scattering of incident energy $\left(I_{0}\right)$ from the central PSF varies as

$$
I_{\text {scattered }} / I_{0}=1-\exp \left[-\left(4 \pi \sigma_{\text {surface }} / \lambda\right)^{2}\right]
$$

the predicted scattering is only $\sim 3 \%$ for a mirror such as theirs, operating at $\sim 300 \AA$. The measured roughness of our mirrors is approximately half of that reported by Spiller et al. Based on these analyses, we conclude that for the MSSTA telescopes, scattering due to surface imperfections should not significantly reduce the fraction of incident energy in the central diffraction peak, and should have a negligible effect on the point spread function. We have determined that the widening of the PSF due to surface scattering by surface imperfections is $\leq 0.1$ arc-second for all MSSTA II telescopes operating at wavelengths $>$ $50 \AA$. For telescopes operating at or below $50 \AA$ scattering begins to play a significant role as the scattered energy increases above $15 \%$ and reduces image quality by increasing the base radius of the encircled energy distribution. Thus, in the case of the small Herschelian $44.1 \AA$ telescope we assumed the scattering resolution error to be $\sim 0.3$ arc-second due to the reduction of the predicted encircled energy distribution of the PSF. In the earlier 1991 flight of the MSSTA I payload, image degradation due to scattering was clearly evident in the spectroheliogram taken at this wavelength (c.f. Allen et al. ${ }^{32}$ ).

\subsection{In-flight Resolution Performance Estimate}

During flight of the MSSTA II payload the SPARCS acquisition and tracking system measured pointing jitter to be $\sigma_{j i t t e r} \sim 0.1$ arc-second. Therefore, if we add the squares of: the pointing jitter error, the resolution focusing error (Eq. 11) and the scattering error due to surface roughness to the theoretical Rayleigh diffraction limit at the wavelength of operation, and then take the square root of this sum, we obtain an upper limit estimate of each telescope's resolution during flight. We define this as the total in-band resolution performance estimate, $\mathcal{R}_{\text {telescope }}(r, \lambda)$, of each telescope operating at wavelength, $\lambda$, at relative radial position $r$ (in the image plane) from the optical axis; given by

$$
R_{\text {telescope }}(r, \lambda) \leq \sqrt{\mathcal{R}_{\text {Rayleigh }}^{2}(r, \lambda)+\varepsilon_{\theta}^{2}\left(r, \lambda_{\text {visible }}\right)+\sigma_{\text {jitter }}^{2}+\sigma_{\text {surface }}^{2}} \leq \mathcal{R}_{m}\left(r, \lambda_{\text {visible }}\right)
$$

We write Eq. (15) as an inequality because $\mathcal{R}_{\text {telescope }}(r, \lambda)$ can in fact be less than the square root argument as it is possible that the resolution focusing error at wavelength, $\lambda$, could be less than that measured at visible wavelengths in our test-target observations; in other words, $\varepsilon_{\theta}(r, \lambda) \leq \varepsilon_{\theta}\left(r, \lambda_{\text {visible }}\right)$. Also, from Eq. 11, values of $\varepsilon_{\theta}\left(r, \lambda_{\text {visible }}\right)$ that result as imaginary in value because the Rayleigh value exceeds the measured visible value (c.f. Table 3 ) are set identically to zero as it is unphysical to have an imaginary angular resolution focusing error as in the case, e.g., of the Ritchey-Chrétien $150 \AA$ telescope which was measured at 0.70 by 0.94 arc-second with a Rayleigh diffraction limit of 1.13 arc-second. This is the bounding condition on $\varepsilon_{\theta}(r, \lambda)$ stipulated by Eq. 11. Hence, as the above calculation is an approximation there is no loss of generality by imposing this condition for the telescopes that operated near or at their visible diffraction-limit resolution. For the latter cases, the resulting in-band resolution performance estimate, $\mathcal{R}_{\text {telescope }}(r, \lambda)$, then simply becomes

$$
\mathcal{R}_{\text {telescope }}(r, \lambda) \leq \sqrt{\mathcal{R}_{\text {Rayleigh }}^{2}(r, \lambda)+\sigma_{\text {jitter }}^{2}+\sigma_{\text {surface }}^{2}}
$$

Using equations (15) and (16) we list in Table 4 the in-band resolution performance estimates for all MSSTA II telescopes during flight 36.091. From the values calculated we estimate that eleven of the nineteen telescopes achieved sub arc-second resolution at their operating wavelength in at least one of two orthogonal angular directions - namely, the RitcheyChrétien: $1216 \AA, 193 \AA, 150 \AA, 284 \AA, 304 \AA$ and $1550 \AA$ telescopes; the Cassegrain $211 \AA$; the Herschelian $211 \AA$ and small Herschelians: $44.1 \AA, 143 \AA$, and $54.7 \AA(\eta)$ telescopes. Based on these estimates, we conclude therefore that the MSSTA II telescopes partially met the mission objective - to perform at sub arc-second resolution. We note however, that the overall performance in some cases was limited to external system variables such as payload pointing jitter and film resolution that affect the quality of the spectroheliograms but not the intrinsic performance of the telescopes. In the years to follow, we expect that such affects will be minimized substantially as technology improves. 


\section{FINAL DISCUSSION \& SUMMARY}

The MSSTA II payload with its compliment of nineteen telescopes was assembled and optically tested at visible wavelengths prior to flight. Five of the two-mirror systems were focused interferometrically at the NASA MSFC. Analysis of their interferograms at the position of best focus returned MTFs which indicate the ability to attain their diffraction-limited resolutions. The Ritchey-Chrétien $1550 \AA$ telescope was focused using a conventional knife-edge test due to the low reflectivity of the coating at $6328 \AA$ used in the interferometric measurements. All telescopes were then aligned and checked for focus at the White Sands Missile Test Range using a Diffraction Limited 16-inch Schmidt-Cassegrain collimator which projected either a pinhole for alignment or a Standard 1951 Air Force High Resolution Test-target for resolution testing. Given this less accurate technique for focusing, all of the Ritchey-Chrétien, Cassegrain and several of the Herschelian telescopes performed admirably well. In the case of the Ritchey-Chrétien telescopes all but one (the $284 \AA$ instrument) were within a factor of $\sim 1-2$ of their visible light diffraction limit. The two Cassegrains were also within a factor of $\sim 1-2$; the $211 \AA$ and $193 \AA$ Herschelians (A and B) were within a factor $\sim 1-2$; and five of the small Herschelians: the $44.1 \AA, 54.7 \AA$ ( $\eta$ ), $143 \AA, 150 \AA(\delta)$, and $284 \AA$ telescopes were within a factor of $\sim 1-2$ of their theoretical values. The remaining small Herschelians' resolution varied widely due to the off-axis alignment of the mirrors coupled with large optic figure errors from mismatching the off-axis mirror segment to the originally designated position, and, from misalignment of the film plane to the optic axis. This was particularly true for the small Herschelians: $54.7 \AA(\beta), 150 \AA(\varepsilon)$, and $180 \AA$; as well as the larger $193 \AA$ Herschelian telescope which performed at $\sim 3-4$ times its theoretical prediction.

In the fabrication and thin-film deposition of some small Herschelian mirrors we encountered some difficulties which prevented us from utilizing the appropriate off-axis mirror originally designated for a particular camera. These offaxis optics were segments cut from a larger, master mirror designed with a $1.0 \mathrm{~m}$ focal length. In order to meet as many of the scientific mission objectives as possible we utilized the off-axis Herschelians that were available and multilayer-coated. The only exception was the $180 \AA$ Herschelian, which on the other hand, being a spherical optic, performs worse off-axis than do the paraboloids. Geometric aberrations and optical misalignment of the mirrors in the dual-mirror telescopes also account for some of the focusing error seen in test-target reproductions. Particularly, it was observed for all telescopes that the resolution in one direction was different than that measured in the orthogonal direction as seen in the test-target images. This effect may have been caused by optical misalignments - most probably, mirror tilt in the dual mirror systems and off-axis misalignment in the single mirror systems - caused during the transport, vibrational load testing and manual handling of the telescopes during system integration with the payload at the White Sands Missile Test Range facility. The difference in calculated resolution versus observed lower resolution at visible wavelengths stems from not utilizing the Rayleigh Criterion as the definitive resolution performance metric and instead measuring the closest spaced peak-to-peak profiles across testtarget line patterns.

Based on an upper limit determination of the resolution focusing errors, as determined by visible light test-target focusing measurements (c.f. Table 3), we have estimated that eleven of the nineteen MSSTA II telescopes performed at inband, sub arc-second resolution during flight; thus partially meeting the mission objective (c.f. Table 4). However, the MSSTA II flight-experiment resulted with only five cameras recording spectroheliograms from six telescopes. Five of the images recorded are useful for analysis but the sixth suffered a pre-filter pinhole contaminating the spectroheliogram with solar visible light. Failures resulting in the other thirteen systems negated the mission performance of those instruments as they did not record any images. The errors attributable to these systems were due to combinations of the following:

i) lack of higher solar flux (nearing solar minimum in year 1994);

ii) transmission filter damage - from vibrational loading and high thermal exposure in the exoatmosphere;

iii) one fractured mirror (193A) - from vibrational loading during launch;

iv) atmospheric absorption - due to Earth's polar tilt relative to the Solar-Earth plane because of the later time of year (November), and, low-Earth altitude (apogee) achieved by the payload;

v) one camera failure;

vi) the improper matching of film to corresponding wavelength observations.

The first five types of system failures are obvious and need no further elaboration. At EUV and soft X-ray wavelengths we used the slower 649 film instead of the faster XUV 100 film to obtain higher resolution images. However, 
this proved to be a mistake: 1) because many telescopes had large focusing errors and could not have obtained such high resolution and 2) the signal-to-noise ratio on the film plane was not large enough to record an image on such slow film. As it is complicated to predict the expected XUV-FUV solar flux during a five minute sounding-rocket experiment it is very difficult to choose with a high level of confidence the correct film to be utilized at each wavelength observed. With the 1991 MSSTA I payload, for example, we successfully recorded a spectroheliogram at $193 \AA$ with a large Herschelian telescope using the higher resolution Kodak 649 film. In 1991, the Sun was near its maximum FUV-XUV intensity; while in 1994 it was diminishing.

\begin{tabular}{|c|c|c|c|c|c|c|c|}
\hline \multicolumn{8}{|c|}{$\begin{array}{l}\text { MSSTA II Telescopes Resolution Performance Estimates } \\
\text { (at respective operating wavelength) }\end{array}$} \\
\hline Telescope & $\begin{array}{c}\lambda \\
(\AA)\end{array}$ & $\begin{array}{c}\text { Measured } \\
\text { Visible Resolution, } \\
\mathbb{R}_{a,}\left(r, \lambda_{\text {visible }}\right) \\
\text { (arc-second) }\end{array}$ & $\begin{array}{c}\text { In-band } \\
\text { Diffraction } \\
\text { Limit, } \\
\text { Reajleigh }(r, \lambda) \text { (arc- } \\
\text { second) }\end{array}$ & $\begin{array}{c}\text { In-band } \\
\text { Resolution } \\
\text { Performance } \\
\text { Estimate, } \\
\text { Recescope }(r, \lambda)(\text { arc- } \\
\text { second) }\end{array}$ & $\begin{array}{l}\text { Measurement } \\
\text { Error }( \pm 1 \sigma) \\
\text { (arc-second) }\end{array}$ & \multicolumn{2}{|c|}{$\begin{array}{c}\text { Film Resolution, Kodak } \\
649 \text { \& XUV-100 } \\
\text { (arc-second) }\end{array}$} \\
\hline \multirow[t]{2}{*}{ Ritchey-Chrétien - I } & 1216 & 1.08 & 0.24 & 0.28 & 0.09 & 0.04 & 0.45 \\
\hline & & 1.79 & $"$ & 1.42 & 0.10 & 0.04 & 0.45 \\
\hline \multirow{2}{*}{ Ritchey-Chrétien - II } & 193 & 1.06 & 0.04 & 0.15 & 0.11 & 0.03 & 0.29 \\
\hline & & 1.37 & $"$ & 0.79 & 0.09 & 0.03 & 0.29 \\
\hline \multirow[t]{2}{*}{ Ritchey-Chrétien - III } & 150 & 0.94 & 0.03 & 0.14 & 0.06 & 0.03 & 0.29 \\
\hline & & 0.70 & $"$ & 0.14 & 0.08 & 0.03 & 0.29 \\
\hline \multirow[t]{2}{*}{ Ritchey-Chrétien - IV } & 284 & 1.24 & 0.06 & 0.53 & 0.06 & 0.03 & 0.29 \\
\hline & & 3.42 & " & 3.24 & 0.41 & 0.03 & 0.29 \\
\hline \multirow{2}{*}{ Ritchey-Chrétien - V } & 304 & 1.12 & 0.06 & 0.15 & $*$ & 0.03 & 0.29 \\
\hline & & 1.12 & $"$ & 0.15 & $*$ & 0.03 & 0.29 \\
\hline \multirow[t]{2}{*}{ Large Herschelian - VI } & 93.9 & 1.65 & 0.02 & 1.21 & 0.21 & 0.10 & 1.03 \\
\hline & & 5.98 & " & 5.88 & 0.24 & 0.10 & 1.03 \\
\hline \multirow[t]{2}{*}{ Ritchey-Chrétien - VII } & 1550 & 1.21 & 0.31 & 0.56 & 0.12 & 0.03 & 0.29 \\
\hline & & 2.94 & " & 2.74 & 0.08 & 0.03 & 0.29 \\
\hline \multirow[t]{2}{*}{ Cassegrain - 1} & 173 & 4.03 & 0.07 & 3.26 & 0.36 & 0.05 & 0.52 \\
\hline & & 5.98 & $"$ & 5.49 & 0.16 & 0.05 & 0.52 \\
\hline \multirow[t]{2}{*}{ Cassegrain - 2} & 211 & 2.07 & 0.09 & 0.17 & 0.19 & 0.05 & 0.52 \\
\hline & & 3.38 & " & 2.41 & 0.19 & 0.05 & 0.52 \\
\hline \multirow[t]{2}{*}{ Herschelian A } & 211 & 1.38 & 0.04 & 0.15 & 0.14 & 0.07 & 0.74 \\
\hline & & 2.58 & $"$ & 2.16 & 0.12 & 0.07 & 0.74 \\
\hline \multirow[t]{2}{*}{ Herschelian B } & 193 & 3.20 & 0.04 & 2.87 & 0.36 & 0.07 & 0.74 \\
\hline & & 4.36 & $"$ & 4.13 & 0.21 & 0.07 & 0.74 \\
\hline \multirow[t]{2}{*}{ Herschelian - $\alpha$} & 44.1 & 3.39 & 0.03 & 0.32 & 0.16 & 0.07 & 0.74 \\
\hline & & 4.57 & " & 2.85 & 0.17 & 0.07 & 0.74 \\
\hline \multirow[t]{2}{*}{ Herschelian - $\beta$} & 54.7 & 26.81 & 0.03 & 26.57 & 0.57 & 0.10 & 1.03 \\
\hline & & 30.94 & $"$ & 30.73 & 0.65 & 0.10 & 1.03 \\
\hline \multirow[t]{2}{*}{ Herschelian $-\gamma$} & 180 & 21.45 & 0.09 & 21.11 & 1.57 & 0.09 & 0.86 \\
\hline & & 17.26 & $"$ & 16.85 & 1.74 & 0.09 & 0.86 \\
\hline \multirow[t]{2}{*}{ Herschelian - $\delta$} & 150 & 5.45 & 0.09 & 4.11 & 0.38 & 0.10 & 1.03 \\
\hline & & 3.92 & $"$ & 1.59 & 0.22 & 0.10 & 1.03 \\
\hline \multirow[t]{2}{*}{ Herschelian $-\varepsilon$} & 150 & 14.23 & 0.09 & 13.77 & 0.35 & 0.10 & 1.03 \\
\hline & & 8.50 & $"$ & 7.71 & 1.13 & 0.10 & 1.03 \\
\hline \multirow[t]{2}{*}{ Herschelian $-\zeta$} & 284 & 4.09 & 0.15 & 1.97 & 0.34 & 0.09 & 0.86 \\
\hline & & 7.63 & $"$ & 6.74 & 0.39 & 0.09 & 0.86 \\
\hline Herschelian $-\eta$ & 54.7 & 3.09 & 0.02 & 0.15 & 0.22 & 0.10 & 1.03 \\
\hline & & 3.51 & $"$ & 0.15 & 0.22 & 0.10 & 1.03 \\
\hline Herschelian - $\theta$ & 143 & 3.39 & 0.04 & 0.17 & 0.16 & 0.07 & 0.74 \\
\hline & & 6.54 & $"$ & 5.47 & 0.38 & 0.07 & 0.74 \\
\hline
\end{tabular}

Table 4. In-band resolution performance estimates during Flight 36.091 as determined by Eq. (5). Estimates are upper-limits which are greater than or equal to the predicted diffraction-limited resolutions for each MSSTA II telescope. The final column lists the film resolution for Kodak 649 (left: 2000 lines $/ \mathrm{mm}$ ) and XUV-100 (right: 200 lines $/ \mathrm{mm}$ ). It can be seen that actual resolution performance is sometimes limited by film resolution. (*: we were unable to locate the resolution test-target film images taken with this telescope and hence, have no measured error for this instrument.)

Nevertheless, of the six telescopes that produced spectroheliograms on the MSSTA II payload, two - the small Herschelian $284 \AA$ and the Cassegrain $173 \AA$ (c.f. Figures 10 and 11 ) - are very good; and one - the Ritchey-Chrétien $1550 \AA-$ 
is spectacular (Figure 9). The short Ritchey-Chrétien $1216 \AA$ telescope produced a reasonable image but with a significant FUV continuum background, demonstrating solar limb darkening (Figure 12). We have not yet measured this telescope's responsivity at its operating wavelength, however we believe that the bandpass of this instrument is wider than we had designed for, possibly $\lambda / \Delta \lambda \sim 8-10$, which might account for this spectroheliogram's observed FUV background continuum. On MSSTA I, the $1216 \AA$ Ritchey-Chretien telescope that was flown produced a very different spectroheliogram that is spectacular in its detail ${ }^{32}$. The $304 \AA$ Ritchey-Chrétien telescope's EUV filter suffered a small pinhole causing visible light leakage onto the film and contaminating the spectroheliogram sufficiently to render the recorded image useless for analysis. Likewise, we discovered that the Cassegrain $173 \AA$ spectroheliogram is also contaminated by scattered light (seen toward the top right-hand corner of the image, c.f. Figure 11), possibly off the telescope's baffle. The Herschelian $180 \AA$ telescope also produced an image, although very faintly (recorded on $649 \mathrm{film}$ ) and very much out of focus as confirmed by the test-target measurements performed at White Sands (Figure 13). As of the time of this writing, the images recorded on this flight are still being analyzed, however, it appears that the Ritchey-Chrétien $1550 \AA$ and $1216 \AA$ telescopes did yield sub arc-second resolution of structures in the chromosphere and transition regions.

The MSSTA was designed for rapid turn around and, with appropriate fixes to problems identified during pre-flight calibrations, vibration testing, launch and landing, could be ready for re-flight in a matter of 12-18 months. With more careful attention and testing devoted to optical alignment and focusing prior to flight, all MSSTA telescopes should achieve sub arc-second performance (limited in some cases by pointing jitter and film resolution). Both flights 36.049 and 36.091 proved to be very successful, and each flight furnished spectacular images showing high spatial resolution of morphology in the Solar atmosphere corresponding to varying details of the physical conditions that produce FUV, EUV and soft X-ray wavelength emissions of plasmas with temperatures ranging from $10^{4}-2 \times 10^{7} \mathrm{~K}$. To date, these images are among the highest spatial resolution quality spectroheliograms ever obtained of the solar atmosphere at these wavelengths.

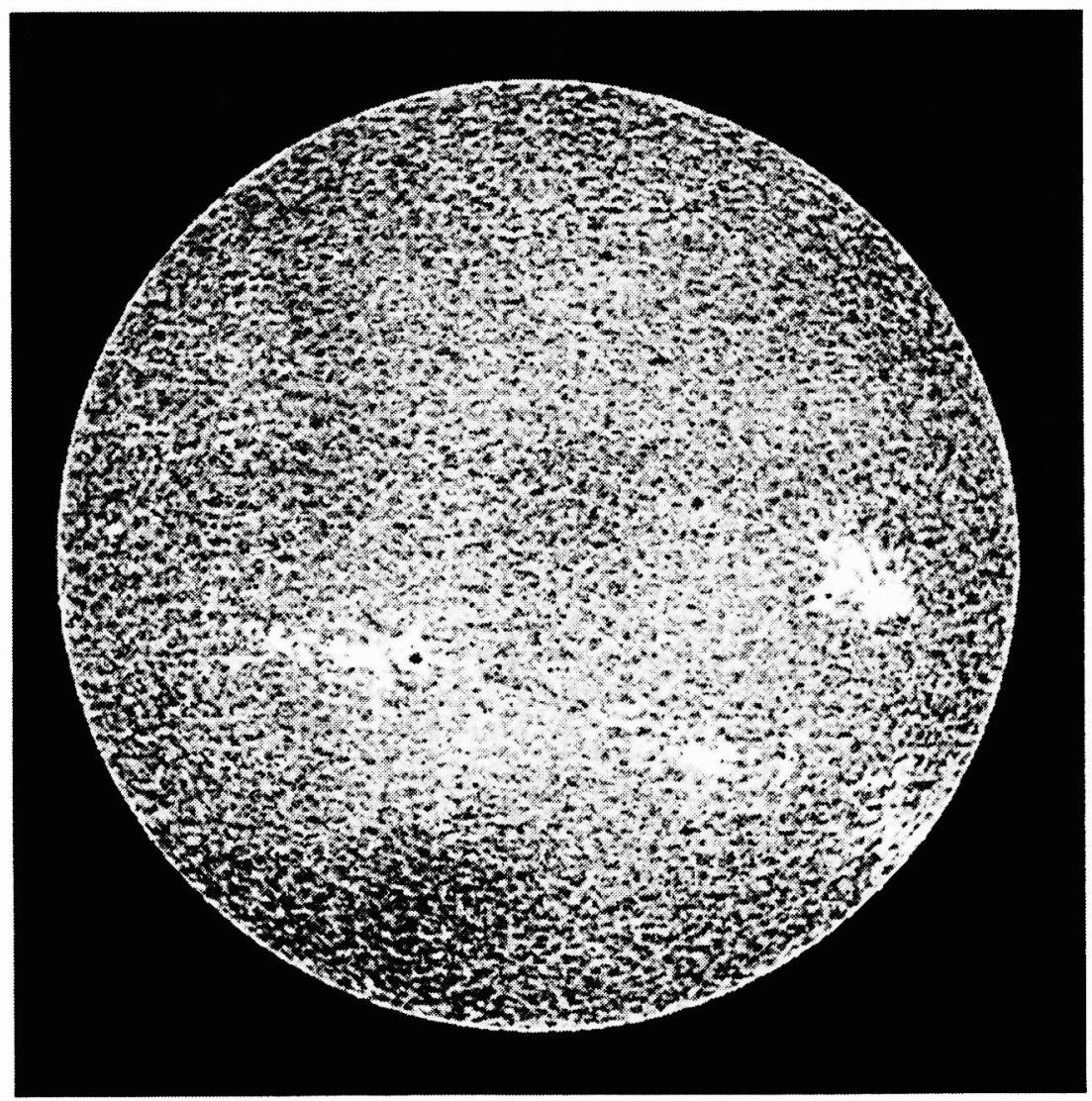

Figure 9. Ritchey-Chrétien $\lambda 1550 \AA$ solar image of C IV plasma in the Transition Region of the solar atmosphere. This plasma is at a temperature, $\mathrm{T} \sim 100,000 \mathrm{~K}$. The image also shows strong FUV continuum due to the rather broad band transmission of this telescope which operates at $\lambda / \Delta \lambda \sim 15$. We are convinced that the bright network boundaries are however, due primarily to C IV emission. Recent Transition-Region and Coronal Explorer (TRACE) images recorded at this wavelength also support this observation, and in the latter case were recorded with a narrower bandpass instrument. This image was recorded on Kodak 649 film. 

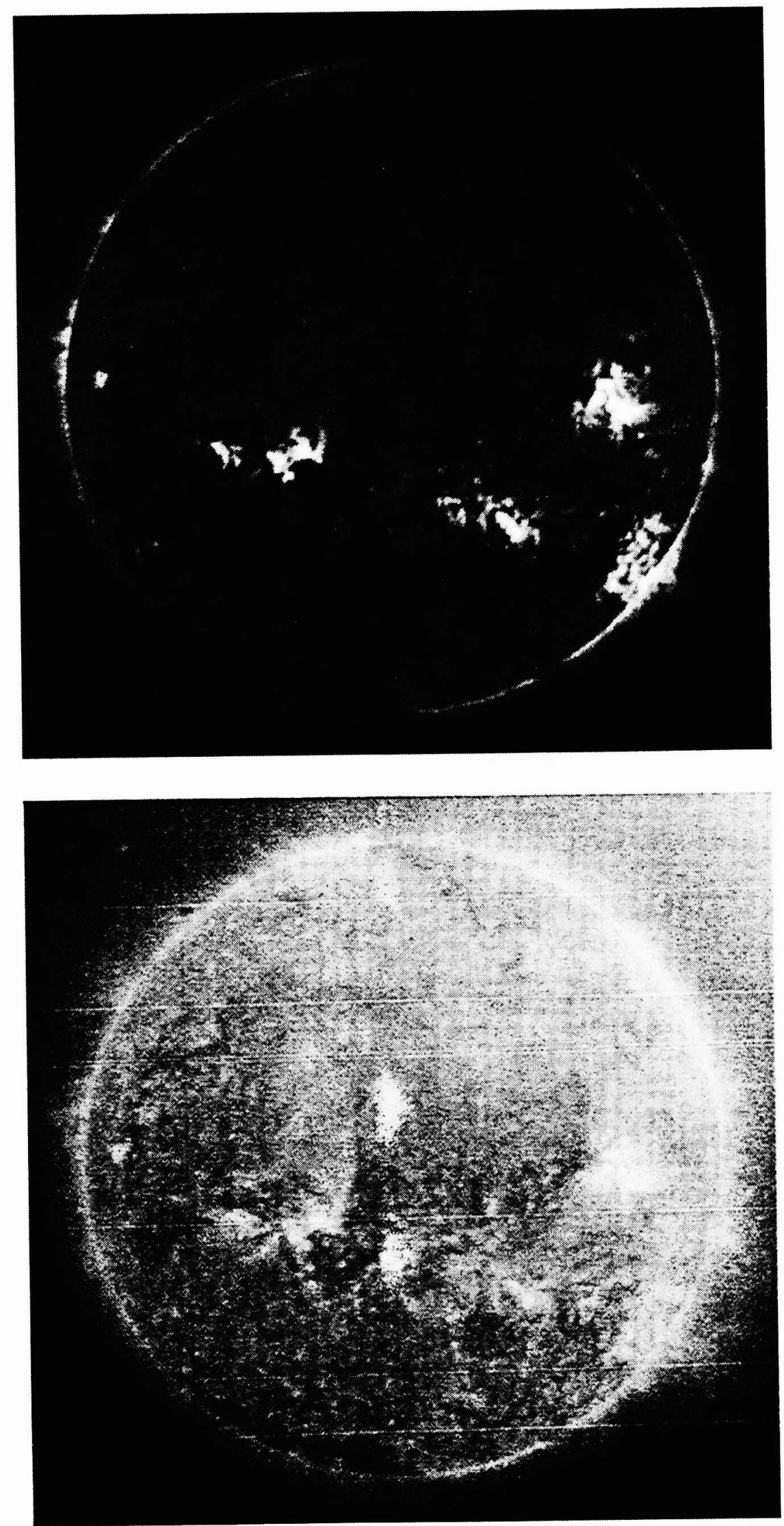

Figure 10. Small Herschelian $2284 A$ solar image of $\mathrm{Fe} X \mathrm{X}$ plasma in the Coronal Region of the solar atmosphere, recorded on Kodak 649 film. This plasma is at a temperature, $\mathrm{T} \sim 3.25 \times 10^{6} \mathrm{~K}$. The image shows very bright active regions. Dimmer "fuzzy-like" structures seen in the darker regions of the image are believed to be contamination of $\lambda 304 \AA$ plasma radiation (He II ions at $\mathrm{T} \sim 80,000 \mathrm{~K}$ ), as the bandpass for this instrument was larger than designed, $\lambda / \Delta \lambda-10$.
Figure 11. Cassegrain $\lambda 173 \AA$ solar image of Fe IX plasma in the Coronal Region of the solar atmosphere. This plasma is at a temperature, $\mathrm{T}$ $6.5 \times 10^{5} \mathrm{~K}$. The image shows very diffuse emission in the quiet (i.e. nonactive) regions of the Sun while is brighter in the active regions, as is likewise observed in the $\lambda 284 \AA$ spectroheliogram of Figure 10. Note the non-linear brightening in the upper right-hand comer of the image which we attribute to scattered light. possibly off the baffle of the telescope. This image was recorded on Kodak XUV-100 film which dries quickly under vacuum and as a result cracks, seen as horizontal striations across the image. 


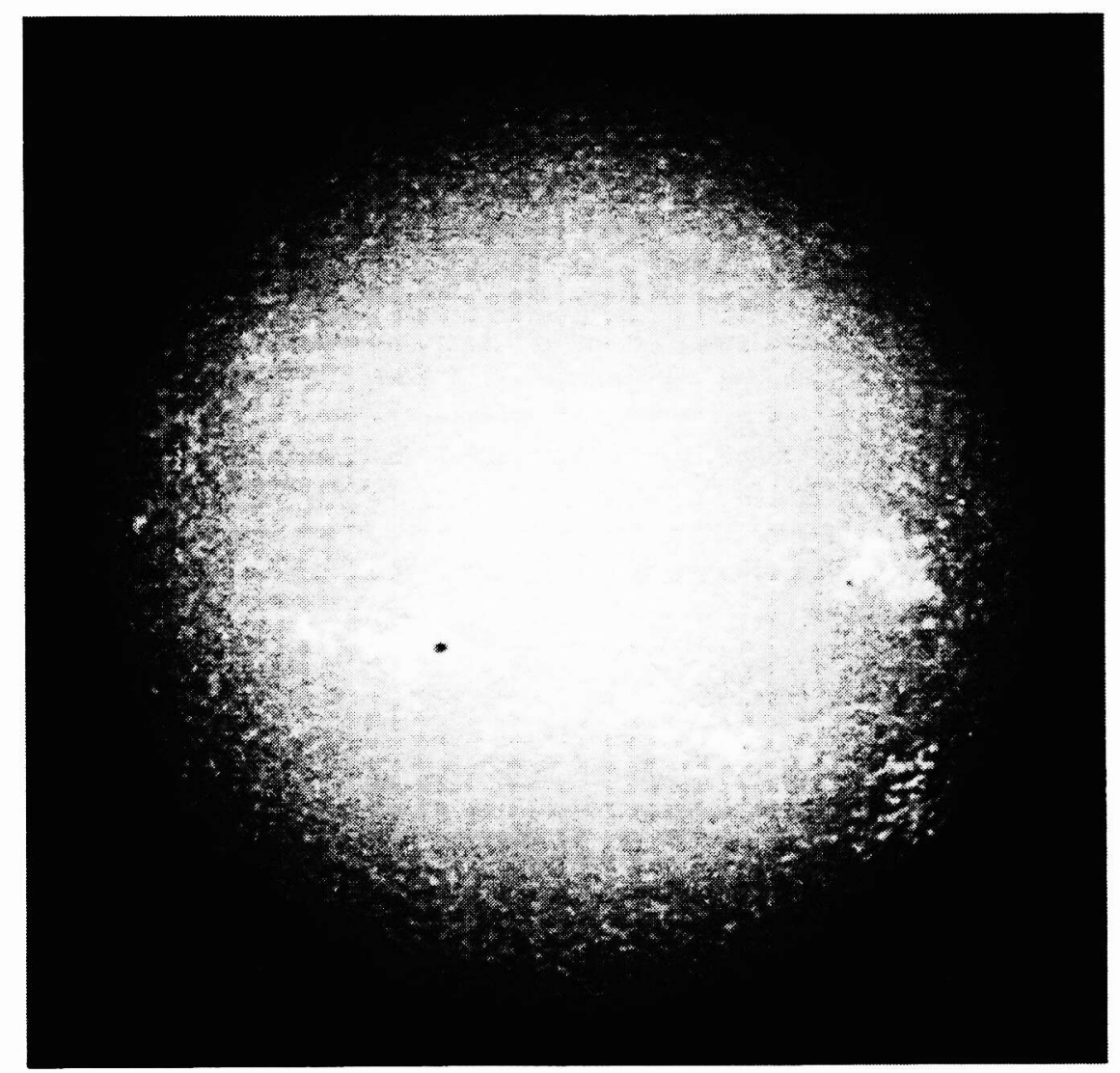

Figure 12. Short Ritchey-Chretien $\lambda 1216 A$ spectroheliogram of the

Chromospheric Region. Lyman-alpha radiation dominates at this bandpass with $\mathrm{H}$ I plasma at a temperature near $\mathrm{T} \sim 20,000-50,000 \mathrm{~K}$. This image was recorded on Kodak 649 film. We believe that the limb darkening seen in this image is due to a wider bandpass of the instrument which leaks continuum background radiation and is optically thick, hence is absorbed more as one progresses toward the limb. It can be seen that there exists bright fine detail on the solar disk corresponding to the network lane structures that are also visible in the C IV image of Figure 10. but in this case is embedded in a bright 'sea' of FUV continuum radiation

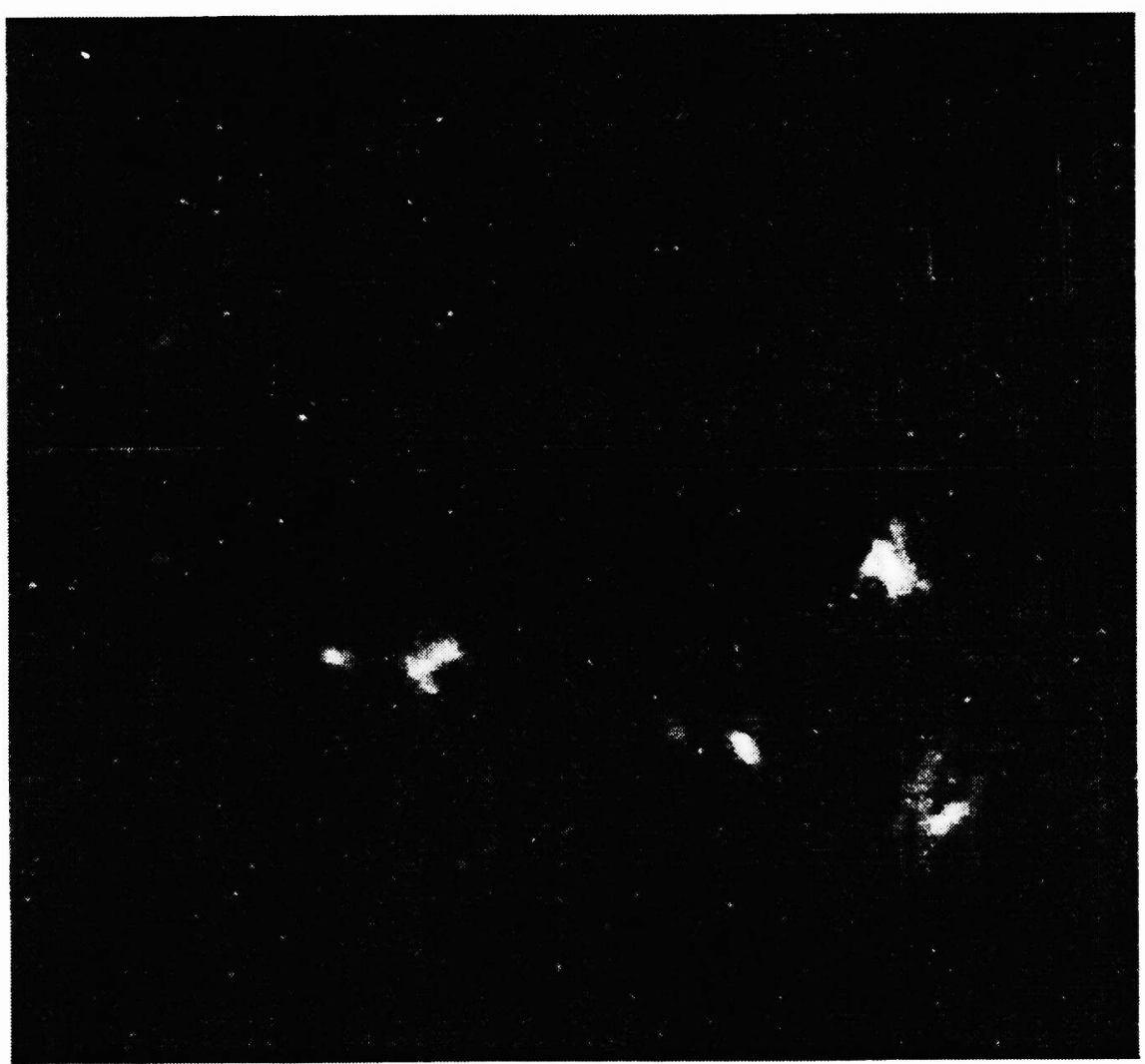

Figure 13. Small Herschellian $\lambda 180 \AA$ solar image of Coronal Region Fe XI plasma at $\mathrm{T} \sim 3.5 \times 10^{6} \mathrm{~K}$. This telescope was grossly out of focus as is evident by the lack of fine detail in this image. Although it is a 150 second exposure, only the bright active regions are observed along with a very faint trace of the solar limb. This image was recorded on the Kodak 649 film which is less sensitive than XUV-100 film at EUV wavelengths. Artifacts such as scratches and dust (from handling) recorded during digitization of the spectroheliogram are clearly evident as the image was digitally enhanced to illustrate the detection of the faint solar limb. 


\section{ACKNOWLEDGMENTS}

This project was funded by NASA Grant NSG-5131, at Stanford University. Additional support was received by Richard Hoover through the NASA Marshall Space Flight Center Director's Discretionary Fund. Troy Barbee, II, was supported by the U.S. Department of Energy through the Lawrence Livermore National Laboratory under contract W-7405Eng-48. Dennis Martínez-Galarce was sponsored by NASA Grant NGT-70284 via the Graduate Student Research Program (GSRP) at Goddard Space Flight Center, and David Gore was supported on NASA GSRP grant NGT-50880 from the Marshall Space Flight Center. We are deeply grateful to Phil Baker of Baker Consulting for the fabrication of superior optical elements, Forbes Powell of Luxel Corporation for his scientific excellence in manufacturing our EUV and XUV filters, Palmer Peters for the multilayer fabrication on the Herschelian $180 \AA$ mirror, and Charlie Welch and the employees of the Lockheed SPARCS team at the White Sands Missile Test Range; without whose expert help we would never have been able to get our payload off the ground. We would also like to thank Brian Hargrave and Walt Fountain for their assistance and use of their laboratory equipment and computers at the Marshall Space Flight Center. Finally, we would like to thank Steve Earls of GND Engineering (formerly SEG Engineering) in Cambridge, MA, for providing a free copy of their final version of the Ektron 1400-series Photoshop® plug-in controller software which allowed us to utilize the Ektron 1412 Digital Camera to successfully digitize all of our images to 12-bit accuracy.

\section{REFERENCES}

1. A. B. C. Walker, Jr., T. W. Barbee, Jr., R. B. Hoover and J. F. Lindblom, "Soft X-ray Images of the Solar Corona with a Normal-Incidence Cassegrain Multilayer Telescope", Science, 241 1781-1787, (1988).

2. L. Golub, G. Nystrom, E. Spiller and J. Wilczynski, "Construction of a multilayered X-ray telescope for solar coronal studies from space", Proc. SPIE, 563, 266-274, San Diego, CA, (1985).

3. L. Golub and M. Herant, "Analysis of the 23 June 1988 flare using NIXT multilayer x-ray images", Proc. SPIE, 1160, 629-635, San Diego, CA, (1989).

4. $\quad$ E. Spiller, "Low-loss reflection coatings using absorbing materials", Applied Physics Letters, 20 365, (1972).

5. E. Spiller, "Multilayer interference coatings for the vacuum ultraviolet", Proc. National Academy of Sciences, Washington, D. C., (1974).

6. E. Spiller, "Reflective multilayer coatings in the far UV region", Applied Optics, 15 2333, (1976).

7. T. W. Barbee, Jr. and D. L. Keith; "Synthetic structures layered on the atomic scale"; Report No. 7804; Workshop on X-ray Instrumentation for Synchrotron Radiation Research; Stanford, CA; Stanford Synchrotron Radiation Laboratory; (1978).

8. T. W. Barbee, Jr., "Multilayer optics for the soft X-ray and extreme ultraviolet", MRS Bulletin, 15 (2), 37-44, (1990).

9. L. Golub, M. Herant, K. Kalata, I. Lavas, G. Nystrom, F. Pardo, E. Spiller and J. Wilczynski, "Sub-arcsecond observations of the solar X-ray corona", Nature, 344 (6269), 842-844, (1990).

10. J. Lindblom, F., A. B. C. Walker, Jr., R. B. Hoover, T. W. Barbee, Jr., R. A. VanPatten and J. P. Gill, "Soft XRay/Extreme Ultraviolet Images of the Solar Atmosphere With Normal Incidence Multilayer Optics", Proc. SPIE, 982, 316-324, San Diego, CA, (1988).

11. J. F. Lindblom, Soft X-ray/Extreme Ultraviolet Images of the Solar Atmosphere with normal incidence multilayer optics, Ph.D. Thesis, Stanford, (1990).

12. E. Spiller, "Normal-Incidence soft X-ray telescopes", Optical Engineering, 30 (8), 1109-1115, (1991).

13. A. B. C. Walker, Jr., J. F. Lindblom, R. H. O'Neal, R. B. Hoover and T. W. Barbee, Jr., "Astronomical observations with normal incidence multilayer optics: recent results and future prospects", Physica Scripta, 41 (6), 1053-1062, (1990).

14. M. J. Allen, First Flight of the Multi-Spectral Solar Telescope Array: Calibration, Results and Future Plans, Ph.D. Thesis, Stanford, (1994).

15. C. E. DeForest, High Resolution Multi-spectral Observations of Solar Coronal Open Structures: Polar and Equatorial Plumes and Rays, Ph.D. Thesis, Stanford, (1995).

16. C. C. Kankelborg, Multispectral Observations of Coronal X-ray Bright Points, Ph.D. Thesis, Stanford, (1996).

17. D. B. Gore, J. B. Hadaway, R. B. Hoover, A. B. C. Walker and C. C. Kankelborg, "Optical Focusing and Alignment of the Multi-Spectral Solar Telescope Array II Payload", Proc. SPIE, 2515, 532-543, San Diego, CA, (1995).

18. A. B. C. Walker, Jr., J. F. Lindblom, R. H. O'Neal, M. J. Allen, T. W. Barbee, Jr. and R. B. Hoover, "Multi-Spectral Solar Telescope Array", Optical Engineering, 29 (6), 581-591, (1990). 
19. R. B. Hoover, P. C. Baker, J. B. Hadaway, R. B. Johnson, C. Peterson, D. R. Gabardi, A. B. C. Walker, Jr., J. F. Lindblom, C. DeForest and R. H. O'Neal, "Performance of the Multi-Spectral Solar Telescope Array. III. - Optical characteristics of the Ritchey-Chretien and Cassegrain telescopes", Proc. SPIE, 1343, 189-202, San Diego, CA, (1991).

20. R. B. Hoover, A. B. C. Walker, Jr., C. E. DeForest, M. J. Allen and J. F. Lindblom, "EUV/FUV response characteristics of photographic films for the Multi-spectral Solar Telescope Array", Optical Engineering, 30 (8), 1116-1124, (1991).

21. T. W. Barbee, Jr., J. W. Reed, R. B. Hoover, M. J. Allen, J. F. Lindblom, R. H. O'Neal, C. C. Kankelborg, C. E. DeForest, E. S. Paris, A. B. C. Walker, Jr., T. D. Willis, E. Gluskin, P. Pianetta and P. C. Baker, "Multi-Spectral Solar Telescope Array II. Soft X-ray/EUV reflectivity of the multilayer mirrors", Optical Engineering, 30 (8), 1067 1075, (1991).

22. C. E. DeForest, C. C. Kankelborg, M. J. Allen, E. S. Paris, T. D. Willis, J. F. Lindblom, R. H. O'Neal, A. B. C. Walker, Jr., T. W. Barbee, Jr., R. B. Hoover and T. W. Barbee, III, "Multi-Spectral Solar Telescope Array V: temperature diagnostic response to the optically thin solar plasma", Optical Engineering, 30 (8), 1125, (1991).

23. M. J. Allen, T. D. Willis, C. C. Kankelborg, R. H. O'Neal, D. S. Martínez-Galarce, C. E. DeForest, L. R. Jackson, J. Lindblom, A. B. C. Walker, Jr., T. W. Barbee, J. W. Weed, R. B. Hoover and F. R. Powell, "Calibration of the multi-spectral solar telescope array multilayer mirrors and XUV filters", Proc. SPIE, 1742, 562-574, San Diego, CA, (1991).

24. M. J. Allen, T. D. Willis, C. C. Kankelborg, R. H. O'Neal, D. S. Martínez-Galarce, C. E. DeForest, L. R. Jackson, J. D. Plummer, A. B. C. Walker, Jr., T. W. Barbee and R. B. Hoover, "Performance of multilayer-coated mirrors for the Multi-Spectral Solar Telescope Array", Proc. SPIE, 2011, 381-390, San Diego, CA, (1993).

25. C. C. Kankelborg, J. Plummer, D. S. Martínez-Galarce, O. N. R. H., C. E. DeForest, A. B. C. Walker, Jr., T. W. Barbee, Jr., J. W. Weed, R. B. Hoover and F. Powell, "Calibration of multilayer mirrors for the Multi-Spectral Solar Telescope Array II", Proc. SPIE, 2515, 436-444, San Diego, CA, (1995).

26. J. Plummer, C. C. Kankelborg, D. S. Martínez-Galarce, R. H. O'Neal, C. E. DeForest, A. B. C. Walker, Jr., R. B. Hoover and T. W. Barbee, Jr., "Design and performance of thin-film XUV filters for the Multi-Spectral Solar Telescope Array II", Proc. SPIE, 2515, 565-575, San Diego, CA, (1995).

27. A. B. C. Walker, Jr., C. E. DeForest, R. B. Hoover and T. W. Barbee, Jr.., "Thermal and Density Structures of Polar Plumes. I. Analysis of EUV Observations with a Multilayer Cassegrain Telescope", Solar Physics, 148 239-252, (1993).

28. A. B. C. Walker, Jr., R. B. Hoover and T. W. Barbee, Jr., "High Resolution Thermally Differentiated Images of the Chromosphere and Corona", Proc. Kluwer Academic Publishers, 83-96, Italy, (1993).

29. C. E. DeForest, A. B. C. Walker, Jr., M. J. Allen, R. B. Hoover and T. W. Barbee, Jr., "The Multi-Spectral Solar Telescope Array IX: Quantitative Measurements of the Solar Corona", Proc. SPIE, 2515, 273-279, San Diego, CA, (1995).

30. C. C. Kankelborg, A. B. C. Walker, II, R. B. Hoover and T. W. Barbee, Jr., "Observation and modeling of soft X-ray bright points. I. Initial Results", Astrophysical Journal, 466 529-536, (1996).

31. C. C. Kankelborg, A. B. C. Walker, II, R. B. Hoover and T. W. Barbee, Jr., "Observation and modeling of soft X-ray bright points. II. Determination of Temperature and Energy Balance", Astrophysical Journal, 491 952-966, (1997).

32. M. J. Allen, H. M. Oluseyi, A. B. C. Walker, II, R. B. Hoover and T. W. Barbee, Jr., "Chromospheric and coronal structure of polar plumes. I. Magnetic structure and radiative energy balance", Solar Physics, 174 367-401, (1997).

33. H. M. Oluseyi, A. B. C. Walker, II, J. Porter, R. B. Hoover and T. W. Barbee, Jr., "Observation and Modeling of the Solar Transition Region - I. A Quasi-static Loop Model with Implications for Heating the Lower Transition Region", submitted to The Astrophysical Journal, (1998).

34. D. S. Martínez-Galarce, A. B. C. Walker, II, R. B. Hoover and T. W. Barbee, Jr., "The Chromosphere/Corona Interface on the Sun. I - Unresolved Coronal Funnels", in preparation, (1999).

35. J. B. Hadaway, R. B. Johnson, R. B. Hoover, J. F. Lindblom and J. Walker, A. B. C., "Design and analysis of optical systems for the Stanford/MSFC Multi-Spectral Solar Telescope Array", Proc. SPIE, 1160, 195-208, San Diego, CA, (1989).

36. A. B. C. Walker, Jr., J. F. Lindblom, J. G. Timothy, R. B. Hoover, T. W. Barbee, Jr., P. C. Baker and F. R. Powell, "High Resolution Imaging with Multilayer Soft x-ray, EUV and FUV telescopes of Modest Aperture and Cost", Proc. SPIE, 1494, 320-333, San Diego, CA, (1991).

37. R. B. Hoover, R. B. Johnson, S. Fineschi, A. B. C. Walker, Jr., P. C. Baker, M. Zukic and J. Kim, "Design and fabrication of the All-Reflecting H-Lyman-alpha Coronagraph/Polarimeter", Proc. SPIE, 1742, 439-451, San Diego, CA, (1992). 
38. P. N. Palmers, R. B. Hoover, R. N. Watts, C. Tarrio and A. B. Walker, "Fabrication of multilayer optics by sputtering: application to EUV optics with greater than $30 \%$ normal reflectance", Proc. SPIE, 2515, 576-581, San Diego, CA, (1995).

39. J. W. Goodman, Introduction to Fourier Optics, McGraw-Hill Publishing Company, San Francisco, (1968).

40. R. N. Bracewell, Two-Dimensional Imaging, Prentice Hall, New Jersey, NJ, (1995).

41. E. L. Dereniak and D. G. Crowe, Optical Radiation Detectors, John Wiley \& Sons, Inc., New York, NY, (1984).

42. G. R. Ayers and J. C. Dainty, "Iterative blind deconvolution method and its application", Optics Letters, 13 (7), $547-$ 549, (1988).

43. G. R. Ayers, "Deconvolution of spatial-correlation spectra for high-resolution astronomical imaging", Optics Letters, 14 (21), 1165-1167, (1989).

44. D. A. Fish, A. M. Brinicombe, E. R. Pike and J. G. Walker, "Blind deconvolution by means of the Richardson-Lucy algorithm", Journal of the Optical Society of America A, 12 (1), 58-65, (1995).

45. D. Kundar and D. Hatizanakos, "Blind Image Deconvolution", IEEE Signal Processing Magazine, 13 (3), $43-64$, (May, 1996).

46. A. H. Bowker and G. J. Lieberman, Engineering Statistics, Prentice-Hall, Inc., Engelwood Cliffs, NJ, (1972).

47. W. P. Zmek, E. C. Moran and J. E. Harvey, "Effects of Surface Quality upon the Performance of Normal Incidence X-Ray/XUV Imaging Systems", Proc. SPIE, 984, 202-212, San Diego, CA, (1988).

48. J. E. Harvey, W. P. Zmek and C. Ftaclas, "Image Quality Prediction of Normal-Incidence X-Ray/EUV Multilayers in the Presence of Substrate and Interface Fabrication Errors", Optical Engineering, 29 603-608, (1990). 